\title{
Christine Rogl
}

\section{SPÄTHELLENISTISCHE APPLIKENKERAMIK UND VERWANDTES AUS EPHESOS Lokale Produktion - Einflüsse - IMPORTe}

Im Folgenden sollen anhand feinkeramischer Beispiele aus späthellenistischen bzw. frührömischen Befunden der Grabungen auf der Tetragonos-Agora einige Aspekte der lokalen Keramikproduktion in Ephesos beleuchtet werden ${ }^{1}$. Ausgewählt wurden dazu applikenverzierte Keramik, Keramik mit Dekor im sog. Westabhang-Nachfolgestil ${ }^{2}$ sowie sog. koisch-knidische Schalen mit appliziertem und/oder gestempeltem Dekor. Dabei sollen in erster Linie Fragen nach den lokalen Produkten, nach eindeutig identifizierten Importen, den wechselseitigen Beeinflussungen, aber auch nach den vorläufigen chronologischen Indizien in Ephesos selbst ${ }^{3}$ und an anderen Fundplätzen gestellt werden.

1 Zusammenfassend zu den Grabungen vgl. P. Scherrer in: D. Parrish (Hrsg.), Urbanism in western Asia Minor, 45. Suppl. JRA (2001) 66-67; ders., ÖJh 63, 1994, Beibl. Grabungen 11-14; ders., ÖJh 65, 1996, Beibl. Grabungen 7-12; ders., ÖJh 66, 1997, Beibl. Grabungen 4-6; ders., ÖJh 67, 1998, Beibl. Grabungen 7-10. Ich danke dem Ausgräber P. Scherrer für Informationen zu den einzelnen Grabungsbefunden. Auch danke ich St. Karwiese für die Auskünfte bezüglich der Münzen, T. Bezeczky bezüglich der Amphoren und A. Giuliani bezüglich der Lampen sowie für weiterführende Diskussion. Auch S. Ladstätter sei für Hinweise und Anregungen zu grundlegenden Definitions- und Begriffsproblemen gedankt. Die Materialaufnahme erfolgte im Zuge des FWF-Projekts 13233 SPR zur hellenistischen Keramik der Tetragonos-Agora von Ephesos durch die Verf. Die meisten Stücke befinden sich im Depot des Österreichischen Grabungshauses. Für die Hilfestellung bei der Aufnahme der Stücke, die sich in der Etütlük-Sammlung des Efes Müzesi Selçuk befinden, sei C. İçten herzlichst gedankt. - Die abgekürzt zitierte Literatur sowie weitere Abkürzungen finden sich am Ende des Beitrags.

2 Beide stehen in bezug auf ihre Formen und den Überzug der pergamenischen Sigillata nahe. Vgl. Meyer-Schlichtmann 1988; Hübner 1993; Behr 1988.

3 Die hier vorgestellten Stücke entstammen Grabungsbefunden, welche die hellenistische bzw. römische Weststoa auf der Tetragonos-Agora betreffen. Diese wurden bisher als späthellenistische Raubgruben bzw. frühkaiserzeitliche Aufschüttungen und teilweise nur als römische Befunde zur Nord- bzw. Südhalle angesprochen, mitunter ist auch die als 'Sentiusschutt' bekannte Auffüllung betroffen. Die Befunde sind derzeit in Bearbeitung. Sie umfassen Material aus dem ausgehenden 2. Jh. v. Chr. bis zum beginnenden 1. Jh. n. Chr. - Raubgrubenfüllungen und frühkaiserzeitliche Platzerhöhungen konnten anhand von Anpassungen als Bestandteile eines einzigen Bauvorganges erschlossen werden. Die enthaltene Keramik in den betreffenden Fundnummern setzt sich zum größten Teil aus späthellenistischen Gattungen und Formen (Reliefbecher, graue runde Ephesos-Platten, Applikenbecher ...) zusammen; diese sind eher kleinteilig und weisen in die Zeit des 1. Drittels des 1. Jhs. v. Chr. Vgl. die Zusammensetzung der Fundkomplexe auf Delos, deren Entstehung mit den Zerstörungen durch Athenodoros $69 \mathrm{v}$. Chr. in Verbindung gebracht wird, z. B. Peignard 1997. Ein weiterer Teil besteht aus Fragmenten von Tellern, Platten und Schälchen in ESA-Qualität sowie Ephesos-Lampen, die mitunter vollständig zusammengesetzt werden können. Dabei sind aber auch einige zeitlich etwas später anzusetzende Formen zu finden, so z. B. spätere ESA-Formen (vgl. Hayes 1985, Form 9, 10, 11, 12, 13B, 14), erstmals ESB-Schälchen (vgl. Hayes 1985, Form 14), Glas-Rippenbecher, dünnwandige Keramik und sog. thorned ware. Die kontextuelle Auswertung läßt die Entstehung bzw. Schließung dieser Raubgruben/Auffüllungen in voraugusteische bzw. augusteische Zeit setzen. Sie können also mit der Erbauung der größeren römischen Agora in Verbindung gebracht werden. Vgl. Hayes 1971, 252-257 Abb. 3-7 ('well' RR/K/60, 20-1 v. Chr.) zu zeitlich entsprechenden Befunden in Knossos oder Hayes 1991, 33 mit für das späte 1. Jh. v. Chr. typischen ESA-Formen aus Paphos. Auch die Münzen der entsprechenden Kisten setzen sich aus meist späthellenistischen und augusteischen Prägungen zusammen. - Von den bisher angesprochenen Befunden ist der oft erwähnte 'Sentiusschutt', welcher ebenfalls in diesem Bereich der Tetragonos-Agora ergraben wurde, aufgrund der im Detail unterschiedlichen Materialzusammensetzung und der offensichtlichen Schadensbrandeinwirkung zu trennen. Vgl. Zabehlicky-Scheffenegger - Schneider 2000, 107 Abb. 1 mit zahlreichen kelchartigen applikenverzierten Gefäßen; ebenda 110-111 zu den ebenfalls enthaltenen ESB-Medaillonschalen sowie, ebenda 111, zum Schadensbrand. Der offensichtliche Unterschied in der Materialzusammensetzung muß in zeitlicher 


\section{Applikenkeramik}

Ausgangspunkt der Untersuchung sind 12 Becherfragmente, welche zur Gattung der sog. Applikenkeramik ${ }^{4}$ bzw. der Firniskeramik mit aufgesetzten Reliefs auf der Wandaußenseite ${ }^{5}$ zählen. Entsprechende Gefäße aus Pergamon werden zwischenzeitlich nicht mehr als eigene Keramikgattung, sondern vielmehr als Untergruppe der formen- und teilweise qualitätsgleichen undekorierten 'pergamenischen Sigillata' betrachtet ${ }^{6}$. Für die applikenverzierten Gefäße wurde bereits früh in der Keramikforschung ein pergamenischer Ursprung vermutet ${ }^{7}$. Sie dürften dort ab der Mitte des 2. Jahrhunderts v. Chr. produziert worden $\operatorname{sein}^{8}$. Im ausgehenden 2. Jahrhundert und besonders im ersten Viertel bzw. der ersten Hälfte des 1. Jahrhunderts v. Chr. findet man sie an zahlreichen weiteren Orten ${ }^{9}$, wo sie in der Folge auch lokal hergestellt wurden ${ }^{10}$. Letzteres scheint auch für die in Ephesos aufgefundenen Stücke der Applikenkeramik zu gelten ${ }^{11}$.

Die meisten der hier vorgelegten Beispiele können aufgrund des Tons und Überzugs (Details s. Katalog) als lokal ephesische Produkte angesprochen werden. Dies wurde bereits für andere Beispiele aus Ephesos bezüglich der Applikenkeramik geäußert ${ }^{12}$ und mittels chemischer Analysen auch nachgewiesen ${ }^{13}$. Im Gegensatz zu den pergamenischen Vergleichsstücken sind der höhere Anteil an Glimmer sowie die oft zu beobachtenden kleinen weißen Einschlüsse hervorzuheben ${ }^{14}$. Eine im Museumsdepot wiederaufgefundene und bisher unpublizierte Matrize (KatNr. 7 Abb. 7-8) für eine Applike mit der Darstellung eines erotischen Symplegma kann hier

Hinsicht interpretiert werden. Der 'Sentiusschutt' wurde bisher in spätaugusteisch/frühtiberische Zeit datiert. Vgl. ebenda 111 mit Anm. 21-22; ähnlich Hayes 1991, 180-186 ('well’ 18, spätaugusteisch-tiberisch). Er könnte mit den Umbau- bzw. Renovierungsarbeiten unter Tiberius und Claudius, die aufgrund der Zerstörungen durch das Erdbeben $23 \mathrm{n}$. Chr. notwendig wurden, in Verbindung gebracht werden. Zu beachten sind dabei auch die enthaltenen ESB-Medaillonschalen. Vgl. Ladstätter 2000, 100. 102-103 mit Anm. 31 und Abb. 7, 1 mit einem späteren Zeitansatz für diese. Auch die Münze M87/31 aus Kiste 87/18 gibt einen Hinweis auf Aktivitäten ab frühestens claudischer Zeit.

4 Zur Definition der Gattung s. Hübner 1993, 1-3; zum Stand der Forschung und der Vorbildfrage, ebenda 4-6. 22-29; zur Technik und Gestaltung, ebenda 30-39. Dabei konnten verschiedene Gefäßformen wie Kannen, Krüge, Amphoren, Askoi, Oinochoen, Kratere, Becher bzw. Skyphoi/Kantharoi, Schalen und auch Lampen mit Appliken versehen werden, ebenda 9-22; s. dazu auch Schwarzer 1999, 298; Bruneau 1991, 600-602.

5 Schäfer 1968, 64-100; Mitsopoulos-Leon 1991, 60-62.

${ }^{6}$ Meyer-Schlichtmann 1988 zur pergamenischen Sigillata; Rotroff 1996, 357. 360 zur Gattungsfrage. Zu beachten sind daher auch die begründeten Einwände von S. I. Rotroff bezüglich der Interpretation G. Hübners zur Applikenkeramik als Kultgeschirr. Vgl. Rotroff 1996, 360; auch Schwarzer 1999, 299.

7 F. Courby, Les vases grecs à reliefs (1922) 449. 477.

8 Vgl. Hübner 1993, 39-49 zu den maßgeblichen 'deposits' in Pergamon (der Große Altar, Bauphasen 12 und 13 des Asklepieions, einige Zisternen in der Stadt) und einer daraus von ihr entwickelten zeitlichen Phaseneinteilung innerhalb der pergamenischen Applikenkeramik, ebenda 49-50 (mit einem Beginn der Produktion in den Jahren 170-160 v. Chr. und einem Auslaufen derselben um die Mitte des 1. Jhs. n. Chr.). Resümee der Datierungsdiskussion und Korrektur der Daten bei Rotroff 1996, 358-360 mit Hinweis auf die genannten pergamenischen 'deposits' sowie zum Spargi-Schiffswrack und dessen Datierungskorrektur in die Jahre 85-75 v. Chr., vgl. Pallarés 1986; E. L. Will, The Spargi Wreck: A reconsideration, AJA 88, 1984, 264; s. auch Warner Slane 1997, 356-357. Zum Beginn der pergamenischen Sigillata allgemein s. Meyer-Schlichtmann 1988, 195-198.

9 Hübner 1993, 4 mit Anm. 10; 95 mit Anm. 39 (letzteres zu Symplegmata-Darstellungen).

10 Hinweise auf Importe bzw. lokale Herstellung s. Hübner 1993, 50-57.

11 Wobei derzeit wohl nicht mit Sicherheit beantwortet werden kann, welches Keramikzentrum des Ostens diese Art der Becher 'erfand'. Auch steht die Diskussion bezüglich der Exporttätigkeiten und -radien sowie möglicher lokaler Imitationen erst am Beginn.

12 Mitsopoulos-Leon 1991, 60.

13 Zabehlicky-Scheffenegger - Schneider 2000, 105. 110 (untersucht wurde das in Abb. 1, 15 bzw. Abb. 2,7 r. o. wiedergegebene Stück). Chemische Analysen ergaben eine Übereinstimmung mit den ephesischen Keramikprodukten wie graue Platten, Reliefbecher und Ephesos-Lampen. Vgl. dazu auch G. Schneider, Chemical and mineralogical studies of Late Hellenistic to Byzantine pottery production in the Eastern Mediterranean, Rei Cretariae Romanae Fautorum Acta 36, 2000, 530-531 Taf. 3.

14 Diese Kalkeinschlüsse verlieren sich bei höheren Temperaturen und können nur mehr als sog. Kalkpseudomorphosen bei vielen der Stücke beobachtet werden. Beachte dabei auch Rotroff 1997, 408-409 Nr. 1650. 1652-1654. 


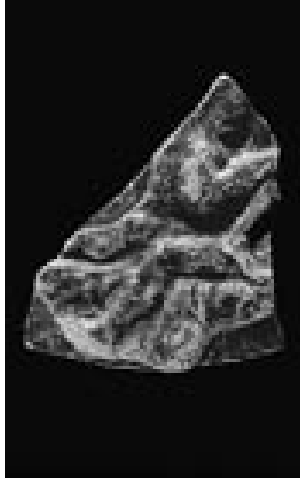

1 KatNr. 1, Inv. AG 87/18-1

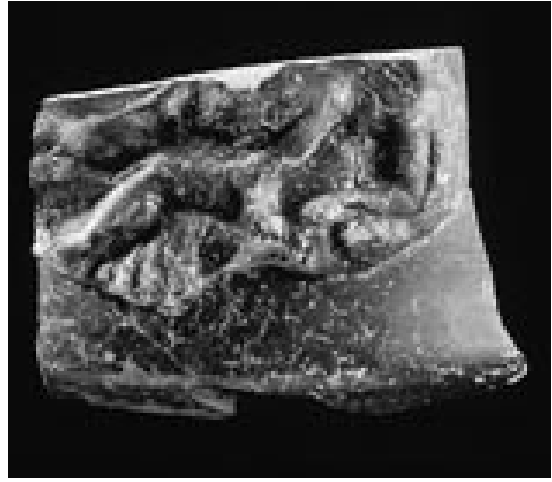

2 KatNr. 2, Inv. AG 95/155-1

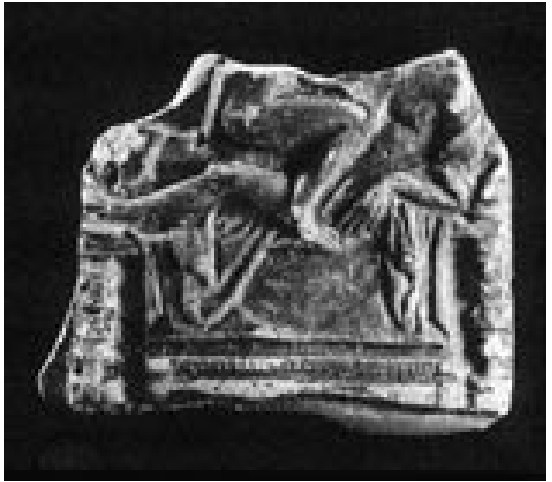

3 KatNr. 3, Inv. AG 96/47-30

eine zusätzliche Bestätigung liefern ${ }^{15}$. Der Firnis dieser applikenverzierten Becher ist außen meist schwarz oder braunrot fleckig und innen sehr oft rot, rotbraun oder orangebraun. Zu betonen ist die sigillataartige, aber oft spröde und wenig glänzende Oberfläche.

Die Profilformen sind mit jenen der pergamenischen Becher ${ }^{16}$ beinahe ident, die Darstellungen und Motive auf den Appliken ebenfalls ${ }^{17}$. Die Formen Hübner Typ I, Form 1-5 bzw. MeyerSchlichtmann S 3 und S 8 scheinen in Ephesos überaus beliebt zu sein ${ }^{18}$. Dabei handelt es sich um breite, gedrungene Becher auf niedrigem profiliertem Fuß. Die Lippe kann nach außen schwingen oder gerade gebildet und innen verdickt sowie abgesetzt sein. Die Darstellungen auf den Appliken lassen den Schluß auf ein großteils auf erotische Symplegmata- (KatNr. 1-7) und Efeu-Korymben-Motive (KatNr. 9-10 Abb. 10) reduziertes Repertoire zu' ${ }^{19}$. Die Liebespaare werden in unterschiedlichen Stellungen wiedergegeben, so z. B. sitzt der weibliche Part meist rittlings auf dem männlichen, kann ihm dabei zugewandt sein (KatNr. 1-3 Abb. 1-3) oder ihm

15 Inv. Et. 774/78 des Efes Müzesi in Selçuk. Der genaue Herkunftsort ist aufgrund fehlender Aufzeichnungen nicht zu eruieren. Zu beachten bleibt allerdings dabei, daß die Verwendung dieses Motivs für Appliken nicht nur auf Becher unseres Typs beschränkt bleibt, sondern auch auf zeitlich etwas späteren, kelchartigen und wohl von der Arretina beeinflußten Gefäßen appliziert wurde. Vgl. Mitsopoulos-Leon 1991, Taf. 71, C 19 mit einem Becherfragment und dazu Zabehlicky-Scheffenegger - Schneider 2000, 109 Abb. 3, 1 r. mit einem Kelchfragment. Zur Entwicklung und zeitlichen Einordnung arretinaähnlicher Typen innerhalb der pergamenischen Sigillata s. MeyerSchlichtmann 1988, 192.

16 Die meist zweihenkeligen Becher werden in der Fachliteratur sowohl als Kantharoi als auch als Skyphoi angesprochen. Sie sind die beliebteste Gefäßform innerhalb der applikenverzierten pergamenischen Sigillata. Vgl. MeyerSchlichtmann 1988, 62-63; er gibt dem Begriff Skyphos den Vorzug.

17 In Ermangelung einer größeren Materialbasis für diese Becher in Ephesos und wegen des schlechten Erhaltungszustandes der präsentierten Fragmente selbst sowie der derzeit nur für Pergamon erarbeiteten Typologie/ Chronologie/Ikonographie wird im Folgenden auf die pergamenischen Ergebnisse zurückgegriffen bzw. werden Vergleiche angestellt. Die Autorin ist sich dabei durchaus bewußt, daß die ephesischen Beispiele, die applikenverzierten wie jene mit Dekor im 'Westabhang-Nachfolgestil', einer Einordnung in die ephesischen Keramikgattungen und Typologien bedürfen.

18 Aufgrund des schlechten Erhaltungszustandes ist eine eindeutige Formzuweisung bei den ephesischen Beispielen mitunter schwierig, so könnte einmal auch die Form B 4 (Mitte/Ende 2. Jh. bis letztes Viertel 1. Jh. v. Chr.) bzw. auch S 1 (Mitte/Ende 2. Jh. bis Ende 1. Jh. v. Chr./Beginn 1. Jh. n. Chr.) ergänzt werden. Vgl. Meyer-Schlichtmann 1988, 78 Taf. 39. 42 (B 4); 63-64 Taf. 39. 43 (S 1).

19 Dieses eklektische Auswählen der Motive könnte ein Hinweis darauf sein, daß man in Ephesos bereits Bekanntes nur imitierte. Auch erinnert es an die beschränkte, beinahe einfallslose Motivwahl bei der in Ephesos hergestellten Keramik mit Dekor im Westabhangstil. Vgl. Mitsopoulos-Leon 1991, Taf. 20-61; Gassner 1997, Taf. 10-13 (meist Efeuranken und sog. Bommelketten). Interessanterweise finden sich aber auch auf den applikenverzierten Bechern im Schwarzmeerraum besonders häufig die Motive der erotischen Symplegmata und der Efeugirlanden. Vgl. Zhuravlev 2000, Taf. 135. 138. Sollte hier daher nicht viel eher an Ephesos als exportierendes Produktionszentrum als an Pergamon gedacht werden? 


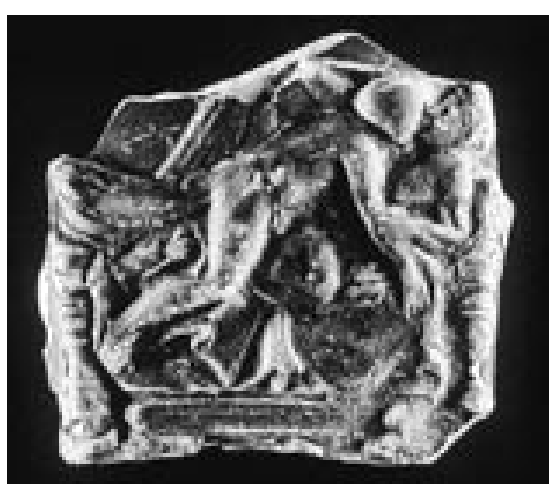

4 KatNr. 4, Inv. AG 92/29-1I

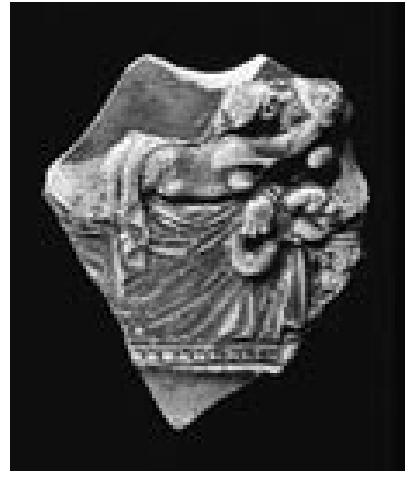

5 KatNr. 5, Inv. AG 89/25, Et. 44, KF $60 / 89$

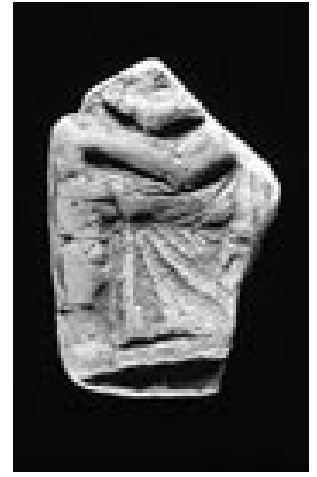

6 KatNr. 6, Inv. AG 95/289-1

auch den Rücken kehren (KatNr. 7 Abb. 7-8). Ebenso kann einer von beiden auf dem Rücken liegend auf der Kline dargestellt sein, der andere liegt dabei auf ihm (KatNr. 5-6 Abb. 5-6). Auch kann der Moment des Besteigens der Kline festgehalten sein (KatNr. 4 Abb. 4). Eine Ausnahme bei den figürlichen Motiven stellt die Präsentation eines Frosches im Inneren eines Bechers dar (KatNr. 8 Abb. 9) ${ }^{20}$. Auffällig ist dabei der profilierte, doch massive Fuß, dessen gesamte Unterseite seine Auflagefläche bildet ${ }^{21}$. Weiters finden sich, wie bereits erwähnt, zwei kleinere Becher mit Efeu-Korymben-Appliken (KatNr. 9-10 Abb. 10) und einmal ein Becher mit Platanen(?)blatt (KatNr. 11 Abb. 11). Letzteres Beispiel ist größer und zeigt ein weiter entwikkeltes Profil als die beiden vorherigen - die Verdickung im Profil der Lippe hat bereits eine Dreiecksform angenommen ${ }^{22}$.

Ein einziges Fragment der ephesischen Beispiele, unsere KatNr. 12 (Abb. 12), stammt von einem Becher der Form Hübner Typ II bzw. Meyer-Schlichtmann B $7^{23}$. Mit dieser Typenbe-

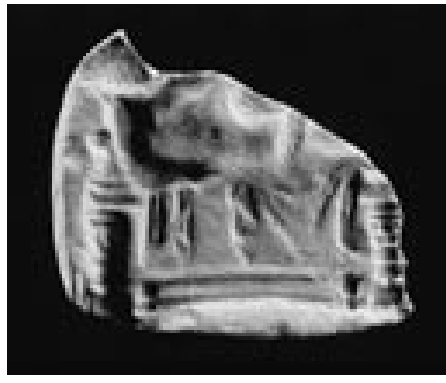

7 KatNr. 7, Applikenmodel, Inv. AG 78/Et. 774

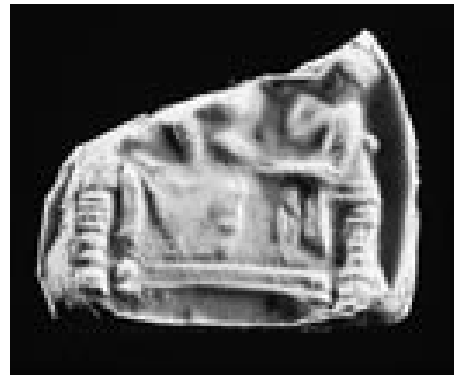

8 KatNr. 7, Silikonabguß

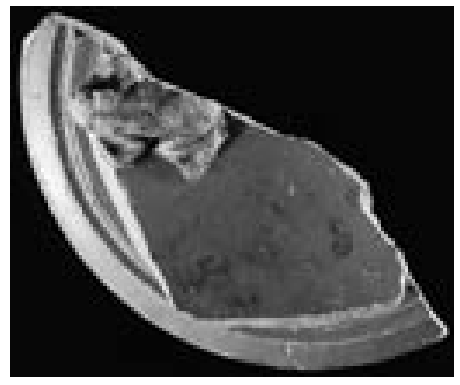

9 KatNr. 8, Inv. AG 93/32-1

20 Da bei diesem Stück weder Glimmer noch die sonst so typischen kleinen weißen Kalkbeimischungen im Scherben beobachtet werden konnten, muß die Frage nach seiner Herkunft vorerst unbeantwortet bleiben. Innenappliken finden sich sonst nicht bei Bechern, sondern sind von den sog. koisch-knidischen Schalen bekannt. Vgl. hier Kap. 3, speziell KatNr. 18. Froschappliken auf Bechern sind nur an wenigen Beispielen, und dann an der Außenseite, angebracht. Vgl. Zhuravlev 2000, Taf. 138, 1; Hübner 1993, Taf. 44, 207.

${ }^{21}$ Vgl. Meyer-Schlichtmann 1988, 70 mit dem Hinweis, daß dieser Entwicklungsschritt an der Form S 8 ab der Mitte des 1. Jhs. v. Chr. beobachtet werden kann.

22 Ebenda 65 (innerhalb des 3. Viertels des 1. Jhs. v. Chr. bis zum Ende der Laufzeit am Beginn des 1. Jhs. n. Chr.).

23 Hübner 1993, 203-204 Abb. 39, 262 Taf. 55-58, 260-271; Meyer-Schlichtmann 1988, 79-80 Taf. 9. 30, 65-67. KatNr. 66 ist dabei mit Appliken versehen. Die Rille könnte ein zeitlicher Hinweis in das 2. Viertel bzw. die Mitte des 1. Jhs. v. Chr. sein. Dieses Stück weist starke Affinitäten (Firnis, Ton) zu pergamenischen Stücken auf. 


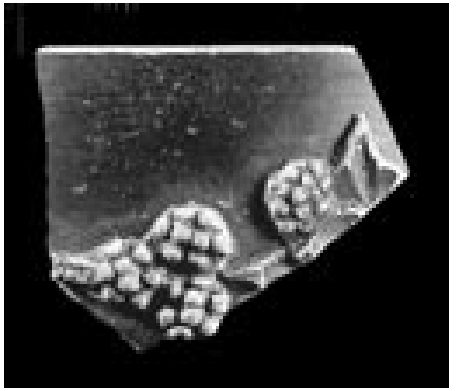

10 KatNr. 9, Inv. AG 89/20, Et. $31, \mathrm{KF} 40 / 89$

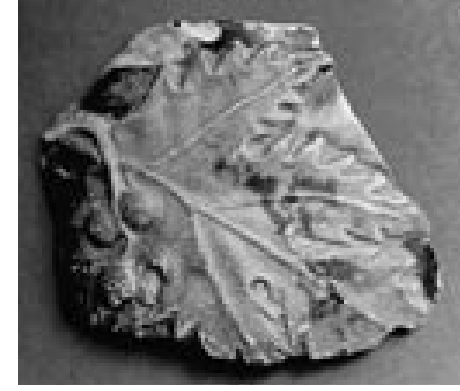

11 KatNr. 11, Inv. AG 90/27-21

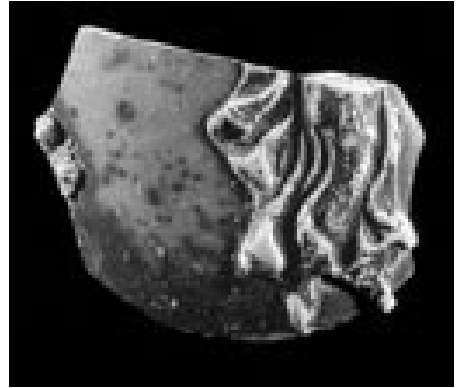

12 KatNr. 12, Inv. AG 90/85-1

zeichnung werden hohe, eher schlanke Becher mit einer Wölbung im Schulterbereich umrissen. Über der Schulter folgen noch eine Einziehung und ein ausschwingender Rand. Das Fragment zeigt den unteren Teil einer Tanzenden, wohl einer Mänade.

Aufgrund des schlechten Erhaltungszustandes unserer Gefäße läßt sich mitunter eine genauere Formzuweisung nicht vornehmen. Auch Henkel haben sich bei ihnen nicht erhalten, dürfen aber in den meisten Fällen angenommen werden ${ }^{24}$.

Bei der Frage nach der zeitlichen Einordnung der zwölf hier präsentierten Fragmente von Applikenkeramik soll zuerst von den bekannten Typen in Pergamon und ihrer zeitlichen Stellung ausgegangen werden. So konnte für die Becherform S 3 in Pergamon eine Laufzeit vom zweiten Viertel des 1. Jahrhunderts v. Chr. bis zum Ende des 1. Jahrhunderts v. Chr. bzw. dem Beginn des 1. Jahrhunderts n. Chr. und für S 8 eine solche von der Mitte/dem Ende des 2. Jahrhunderts v. Chr. bis zum dritten Viertel des 1. Jahrhunderts v. Chr. erarbeitet werden ${ }^{25}$. Eine Änderung in der Lippenausbiegung sowie in der Gestaltung des Fußes von S 8 stellt eine weitergeführte Variante dieses Typs bis ins letzte Drittel des 1. Jahrhunderts v. Chr. dar.

Weitere Anhaltspunkte in chronologischer Sicht bietet Delos, und hier vor allem jene Fundkomplexe, die mit den Zerstörungen einerseits durch Mithridates 88 v. Chr. und andererseits durch Athenodoros 69 v. Chr. verknüpft werden. Diese liefern uns eine Übersicht über das Keramikspektrum der Zeit des ersten und beginnenden zweiten Viertels des 1. Jahrhunderts v. Chr. Darin sind bereits Becher des Typs Meyer-Schlichtmann S 1 bzw. S 8 vertreten ${ }^{26}$. Es müssen jedoch nicht, wie bisher vermutet, alle Beispiele der Applikenkeramik auf Delos ${ }^{27}$ pergamenische Importe sein ${ }^{28}$. Auch Töpfereien in Ephesos und der umgebenden Region könnten ihre Produkte dahin exportiert haben. Anhaltspunkte, die ins erste bzw. zweite Viertel des 1. Jahrhunderts v. Chr. fallen, bieten uns noch ein Komplex in Tel Anafa sowie das Schiffswrack von Spargi ${ }^{29}$.

In Athen findet sich Applikenkeramik in geringer Zahl in Kontexten des 1. Jahrhunderts v. Chr. Diese ist mit Sicherheit nicht lokal hergestellt, sondern aus dem Osten importiert. Dabei

24 Dabei handelt es sich meist um sog. (vertikale) Rotellenhenkel. Vgl. Meyer-Schlichtmann 1988, Taf. 7, 1 (S 1). Es können allerdings auch vertikale Ringhenkel mit Daumenplatten angebracht sein; vgl. ebenda 13 (S 3); Ladstätter - Lang-Auinger 2001, 77-78 Taf. 51, 54 (ein Beispiel aus dem Hanghaus 2 in Ephesos).

25 Meyer-Schlichtmann 1988, 65 Taf. 7. 40 (S 3); 69-70 Taf. 8. 39 (S 8). Ähnliche Laufzeiten ergeben sich für die Formen B 4 und S 1.

${ }^{26}$ A. Peignard-Giros in: 5. Kongreß zur hellenistischen Keramik, Chania 1997 (2000) 134 mit Anm. 18 sowie Taf. 80, 2; Chatzidakis 2000, 120 Abb. 3, 28. Beachte all die verschiedenen Formen bei Bruneau 1991, 644 ff., die im 1. Viertel des 1. Jhs. v. Chr. auf Delos in Gebrauch stehen.

27 Bruneau 1991, 609.

28 Bereits Hübner 1993, 57 stellte Unterschiede zwischen der pergamenischen Applikenkeramik und jener, die auf Delos gefunden wurde, fest. Eine relativ geringe Übereinstimmung bezüglich der Motive beobachteten auch Zabehlicky-Scheffenegger - Schneider 2000, 110 und Ladstätter u. a. 2003, 29 mit Anm. 97.

29 Warner Slane 1997, 358 Taf. 30, FW 499 (Hell 2B/C »deposit« bis 75 v. Chr.); Pallarés 1986, 95-96 Taf. 7e. 
handelt es sich meist um Becher mit Efeuappliken. Die meisten wurden als pergamenische Importe identifiziert, andere ließen jedoch keine nähere Herkunftsbestimmung $\mathrm{zu}^{30}$.

Für Ephesos selbst liegen inzwischen mehrere aufgearbeitete Befunde vor. Zu nennen sind neben den hier präsentierten Fragmenten aus den sog. späthellenistischen Raubgruben und frühkaiserzeitlichen Aufschüttungen sowie aus nicht näher determinierten römischen Planierungsbefunden der Tetragonos-Agora ${ }^{31}$ vor allem mehrere Brunnenfüllungen. Brunnen SR 9c in Hanghaus 1 enthielt in Füllung 1 und 2 jeweils ein Beispiel der Applikenkeramik ${ }^{32}$. Damit gewinnen wir ein Kontextdatum von 100-25 v. Chr. für diese zwei Beispiele. Für die Becher aus dem Brunnen am Staatsmarkt, die aus Füllung A bzw. B1 stammen, konnte nur eine grobe Zuweisung in späthellenistische/augusteische Zeit bzw. ins erste Viertel des 1. Jahrhunderts n. Chr. vorgenommen werden ${ }^{33}$. Beide für uns relevanten Stücke gehören zu Meyer-Schlichtmanns Form S 8 und zeigen einerseits ein erotisches Symplegma (Besteigen der Kline), andererseits eine Efeugirlande. Auch sie dürften lokal ephesische Produkte und keine Importe aus Pergamon sein. Aus der Verfüllung der Drainage im Bereich des Südtores der Tetragonos-Agora wurden explizit keine Beispiele bekanntgemacht, jedoch sind zwei Gefäßprofile abgebildet, die an unsere Becher erinnern $^{34}$. In einem Fundkomplex eines hellenistischen Niveaus in Hanghaus 2 findet sich ein einziges Exemplar der Daumenplatte eines Henkels von einem Applikenbecher. Dieser Komplex umfaßt Keramik der Zeitspanne 170-130 v. Chr., das Enddatum könnte aufgrund dieses Henkels eventuell geringfügig nach unten gesetzt werden ${ }^{35}$.

Resümierend bedeutet dies für die Datierung der hier präsentierten Becherformen der Applikenkeramik, daß sowohl ephesische Fundkomplexe als auch außer-ephesische Vergleiche eine zeitliche Einordnung bzw. Laufzeit vom Ende des 2./Beginn des 1. Jahrhunderts v. Chr. bis zum Ende des 1. Jahrhunderts v. Chr./Beginn des 1. Jahrhunderts n. Chr. erlauben ${ }^{36}$. Eine Konzentration dürfte dabei im ersten und zweiten Viertel des 1. Jahrhunderts v. Chr. liegen.

KatNr. 1 (Abb. 1 Taf. 1, 1)

Inv. AG 87/18-1

Befund: frühkaiserzeitliche Aufschüttung, römische Agora (M 87/23-31)

Gattung/Form: Applikenbecher; Hübner Typ I, Form 1; Meyer-Schlichtmann S 8

DmR ca. 13 cm; H 4,6 cm; B 4,2 cm; Ws 0,2-0,25 cm

Ton: 5YR6/6 reddish yellow (braunorange); hart; sehr fein, kaum weiße Beimischungen, Glimmer.

Firnis: 5 YR3/1 bis $3 / 2$ very dark gray bis dark reddish brown (schwarzbraun); leicht glänzend; gut haftend.
Erhaltung: Wand/Applike.

Dekor: Erotisches Symplegma auf Kline: Die weibliche Figur hockt rittlings auf der männlichen, dieser zugewandt; am rechten Oberschenkel sind Reifen auszumachen, der linke Unterschenkel des Mannes fällt mit den Stoffbahnen von der Kline herab, diese wird nur angedeutet.

Vgl./Dat.: Form: Meyer-Schlichtmann 1988, 65 Taf. 8. 39 (S 8, Mitte/Ende 2. Jh. - Ende 1. Jh. v. Chr./Beginn 1. Jh. n. Chr.); Hübner 1993, Abb. 5. 7; Applike: Hübner 1993, 195 Taf. 30, 151 (ähnelt unserem Beispiel); 28, 139

30 Rotroff 1997, 222. 408-409. 1650. 1652-1654. Die Tonbeschreibungen jener nicht sicher Pergamon zugewiesenen Fragmente erinnern an ephesische Produkte; erwähnt werden der hohe Glimmergehalt und mitunter die zahlreichen weißen Einschlüsse.

31 Vgl. Anm. 3.

32 Ladstätter u. a. 2003, 24, Taf. 2, K 15 (Randfrgt. mit verdickter und abgesetzter Lippe innen, S 3) aus Brunnenfüllung 1 (50-25 v. Chr.) und 29 Taf. 7, K 67 (Wandfrgt., Form [?], Applike mit Eros/Flötenspieler [?]) aus Brunnenfüllung 2 (ca. 100 v. Chr.).

${ }^{33}$ Meriç 2002, 30-31 Taf. 5. 91, K 37-38. Chronologisch interessant ist dabei das bei ihm folgende Stück K 38a, ein kelchartiges, applikenverziertes Gefäß aus Füllung B 3, welche in die Zeit 50-75 n. Chr. datiert wird; vgl. o. Anm. 3 .

34 Gassner 1997, 48-50 Taf. 7, 114-115. Diese werden von ihr ins letzte Drittel des 2. Jhs. v. Chr. datiert.

35 Ladstätter - Lang-Auinger 2001, 77-78 Taf. 51, 54.

36 Interessant zu untersuchen wäre dabei auch die unterschiedliche Gestaltung der Appliken, z. B. bei den erotischen Symplegmata. Warum sind manche mit detailreichster und manche mit beinahe schmuckloser Kline gestaltet? Könnte hier ein chronologischer Hinweis enthalten sein? Vorläufig sind aber aus Ephesos zu wenige und vor allem zu wenige vollständige Exemplare bekannt, um hierüber Anhaltspunkte zu erhalten. 
(Reif am Oberschenkel); Mitsopoulos-Leon 1991, 60-61. 64 Taf. 70, C18; Bruneau 1970, Taf. 41, D 26-27; Bruneau 1991, 636, 64.

KatNr. 2 (Abb. 2 Taf. 1, 2)

Inv. AG 95/155-1

Befund: römische Weststoa (M 95/41?)

Gattung/Form: Applikenbecher; Hübner Typ I, Form 2-5; Meyer-Schlichtmann S 3 bzw. B 4

DmR ca. $12 \mathrm{~cm}$; H 4,3 cm; B 5,4 cm; Ws 0,3 cm

Ton: 5YR5/6 yellowish red (rotorange); sehr hart; sehr fein, wenige weiße Beimischungen, Glimmer.

Firnis: a: 10R3/1 dark reddish gray (schwarzbraun); i: 2.5YR4/4 reddish brown (rotbraun); leicht glänzend; gut haftend.

Erhaltung: Wand/Applike.

Dekor: Erotisches Symplegma auf Kline: Die weibliche Figur hockt rittlings auf der männlichen, dieser zugewandt; der linke Unterschenkel des Mannes fällt mit den Stoffbahnen von der Kline herab; diese wird nur angedeutet.

Vgl./Dat.: Form: Meyer-Schlichtmann 1988, 65 Taf. 7 40 (S 3, 2. Viertel 1. Jh. v. Chr. - Ende 1. Jh. v. Chr./ Beginn 1. Jh. n. Chr.); 78 Taf. 39. 42, B 4 (Mitte/Ende 2. Jh. - 4. Viertel 1. Jh. v. Chr.); Hübner 1993, Abb. 5-6; Applike: KatNr. 1; Schäfer 1968, 80 Taf. 29, E 35 (IIIc).

Publiziert: Zabehlicky-Scheffenegger - Schneider 2000, 107 Abb. 1, 16.

KatNr. 3 (Abb. 3 Taf. 1, 3)

Inv. AG 96/47-30

Befund: hellenistische Weststoa/Nord 1 - Raubgrube (M 96/21-27)

Gattung/Form: Applikenbecher; Hübner Typ I, Form 2-5; Meyer-Schlichtmann S 3

DmR ?; H 4,2 cm; B 5,3 cm; Ws 0,25-0,3 cm

Ton: Becher: rotorange; hart; feine weiße Beimischungen; Applike: orangebeige; mittelhart; feine weiße Beimischungen, Glimmer (?)

Firnis: a: schwarz; i: rotbraun; eher matt; gut haftend. Erhaltung: Wand/Applike.

Dekor: Erotisches Symplegma auf Kline: Die weibliche Figur hockt rittlings auf der männlichen, dieser zugewandt; Kline sehr aufwendig gestaltet, sehr detailreich.

Vgl./Dat.: Meyer-Schlichtmann 1988, 65 Taf. 7. 40 (S 3, 2. Viertel 1. Jh. v. Chr. - Ende 1. Jh. v. Chr./Anfang 1. Jh. n. Chr.); Hübner 1993, Abb. 5-6; Applike: Hübner 1993, 195 Taf. 30, 151 (sehr ähnlich).

KatNr. 4 (Abb. 4 Taf. 1, 4)

Inv. AG 92/29-1

Befund: hellenistische Weststoa/Nord 2 - Raubgrube, frühkaiserzeitliche Aufschüttung (M 92/18-23)

Gattung/Form: Applikenbecher; Hübner Typ I, Form 1; Meyer-Schlichtmann S 8 (?)

DmR ?; H 4,7 cm; B 5,2 cm; Ws $0,3 \mathrm{~cm}$

Ton: 7.5YR6/6 reddish yellow (braunorange); hart; sehr fein, Glimmer (?).

Firnis: a: 7.5YR3/2 dark brown (braun); i: 2.5YR4/6 dark red (rotbraun); eher matt; gut haftend.
Erhaltung: Wand/Applike.

Dekor: Liebespaar im Moment vor dem Zusammenkommen: Die nackte männliche Figur besteigt die Kline, die weibliche Gestalt, die auf der Kline ruht, zieht den Mann zu sich; Kline aufwendig gestaltet.

Vgl./Dat.: Form: Meyer-Schlichtmann 1988, 69-70 Taf. 8. 39. 42 (S 8, Mitte/Ende 2. Jh. - 1. Viertel 1. Jh. v. Chr./bes. 3. und 4. Viertel 1. Jh. v. Chr.); Hübner 1993, Abb. 5; Applike: Hübner 1993, 194 Taf. 29, 144 (Stoffe fehlen unter der Kline); Meric 2002, 31 Taf. 5. 91, K 37; Schäfer 1968, 80 Taf. 32, E 55 (VI); Zhuravlev 2000, Taf. 135, 4.

KatNr. 5 (Abb. 5 Taf. 1, 5)

Inv. AG 89/25-1 bzw. Et. 44, KF 60/89, Efes Müzesi Selçuk

Befund: römische Agora

Gattung/Form: Applikenbecher; Hübner Typ I, Form 1; Meyer-Schlichtmann S 8

DmR ca. $15 \mathrm{~cm}$; H 5,4 cm; B 4,8 cm; Ws 0,2-0,35 cm

Ton: rotbraun; sehr hart; sehr fein, wenige weiße Beimischungen, Quarz, wenig Glimmer.

Firnis: a: $2.5 \mathrm{YR} 3 / 6$ bis $5 \mathrm{YR} 3 / 3$ dark red bis dark reddish brown (rot bis braunschwarz); i: 2.5YR4/8 red (orangebraun); glänzend; gut haftend.

Erhaltung: Wand/Applike.

Dekor: Erotisches Symplegma auf Kline: Die weibliche Figur liegt auf der gelagerten männlichen, dieser zugewandt, sie umfaßt mit der rechten Hand den Kopf des Mannes und küßt ihn; der Mann hält einen Kranz (Epithymis); die unteren Körperregionen sind vom Klinentuch bedeckt; Kline detailreich gestaltet.

Vgl./Dat.: Form: Meyer-Schlichtmann 1988, 69-70 Taf. 8. 39. 42 (S 8, Mitte/Ende 2. Jh. v. Chr. - Ende 1. Jh. v. Chr./Beginn 1. Jh. n. Chr.); Hübner 1993, Abb. 5. 7; Applike: Hübner 1993, 194 Taf. 29, 147 (Bildtypus entspricht); Mitsopoulos-Leon 1991, C18b (ähnlich; Kranz, Hetäre anders); Kranz vgl. Schäfer 1968, Taf. 31, E 48; Bruneau 1991, 636, 65

KatNr. 6 (Abb. 6 Taf. 1, 6)

Inv. AG 95/289-1

Befund: römische Weststoa (M 95/57-58)

Gattung/Form: Applikenbecher; Hübner Typ I, Form 1; Meyer-Schlichtmann S 8

DmR ?; H 3,55 cm; B 2,5 cm; Ws 0,2-0,4 cm

Ton: 5YR7/4 pink (rosabeige); mittelhart; fein, kaum weiße Beimischungen, Glimmer.

Firnis: a: 2.5 YR5/6 red (braunrot); i: 2.5 YR $4 / 8$ red (rotbraun); eher matt; gut haftend, aber berieben.

Erhaltung: Wand/Applike.

Dekor: Erotisches Symplegma auf Kline, teilweise erhalten: Von der auf dem Rücken liegenden weiblichen Figur mit angezogenen Beinen und der auf ihr liegenden männlichen haben sich nur ein Fuß bzw. ein Bein erhalten.

Vgl./Dat.: Form: Meyer-Schlichtmann 1988, 69-70 Taf. 8. 39. 42 (S 8, Mitte/Ende 2. Jh. v. Chr. - Ende 1. Jh. v. Chr./Beginn 1. Jh. n. Chr.); Hübner 1993, Abb. 5. 7; Applike: Hübner 1993, 194 Taf. 28, 139; 195 Taf. 31, 152b.1 (Bildtypus entspricht); Schäfer 1968, 80 Taf. 27, E 23 (IIa); Bruneau 1991, 635, 63; Zhuravlev 2000, Taf. 135,5 . 
Publiziert: Zabehlicky-Scheffenegger - Schneider 2000, 109 Abb. 3, 2 linkes Fragment.

KatNr. 7 (Abb. 7-8 Taf. 1, 7) Inv. Et. 774/78, Efes Müzesi Selçuk

Befund/Herkunft: nicht gesichert, vielleicht Grabungen 1978 im Osten der Agora (Information P. Scherrer)

Gattung/Form: Applikenmodel

H 4,2 cm; B 4,9 cm; Ws 0,9-1,2 cm

Ton: 7.5YR7/6 reddish yellow (beigeorange); sehr hart; sehr fein, kleinste weiße Beimischungen, Glimmer.

Firnis: -

Erhaltung: rechter oberer Teil abgebrochen.

Dekor: Erotisches Symplegma auf einer Kline: Die nackte weibliche Gestalt hockt rittlings auf dem Mann, wendet ihm dabei ihren Rücken zu; insgesamt sehr detailreich gestaltet.

Vgl./Dat.: Hübner 1993, 194-195 Taf. 27-32; ebenda Taf. 29, 143 (laut Katalogtext ist die Frau auf den übrigen pergamenischen Beispielen aufrechter wiedergegeben); Mitsopoulos-Leon 1991, 61. 64 Taf. 71, C19; Schäfer 1968, 80 Taf. 30, E 44-46 (IVa-b); das Motiv entspricht dabei Zabehlicky-Scheffenegger - Schneider 2000, 109 Abb. 3, 1; zu erotischen Szenen s. auch Bruneau 1991, 605-606.

KatNr. 8 (Abb. 9 Taf. 1, 8)

Inv. AG 93/32-1

Befund: hellenistische Weststoa/Nord 1+2 - Raubgrube (M 93/23)

Gattung/Form: Applikenbecher; Hübner Typ I oder III, Form 11 (?); Meyer-Schlichtmann S 8 (spät)

DmB 8,0 cm; H 1,5 cm; B 7,3 cm; Ws 0,3-0,4 cm

Ton: 5YR6/8 reddish yellow (orangerosa); hart; sehr fein. Firnis: 2.5YR6/8 light red (orangebraun); sigillataartig glänzend; gut haftend.

Erhaltung: Standring/Boden.

Dekor: Froschapplike, detailreich, Frosch ca. 2,1 cm lang.

Vgl./Dat.: Form: Hübner 1993, Abb. 14, K 112; MeyerSchlichtmann 1988, 69-70 Taf. 8. 39. 42 bes. Taf. 29, 29 (S 8, ab der Mitte des 1. Jhs. v. Chr.); Applike: Hübner 1993, 32. 153. 191 Taf. 15, Motiv 91 bzw. Taf. 44, 207 (außen, kombiniert mit Silensapplike); zu Tierappliken allgemein, ebenda 49.

KatNr. 9 (Abb. 10 Taf. 1, 9)

Inv. AG 89/20-1 bzw. Et. 31, KF 40/89, Efes Müzesi Selçuk

Befund: hellenistische Weststoa/Nord 3 - Raubgrube

Gattung/Form: Applikenbecher; Hübner Typ I, Form 2-5; Meyer-Schlichtmann S 3

DmR 9,8 cm; H 4,0 cm; B 4,8 cm; Ws 0,3 cm

Ton: rot bis orangerot; sehr hart; sehr fein, kaum weiße Beimischungen, wenig Glimmer.

Firnis: a: 7.5 YR-N3 bis N2 very dark gray bis black (schwarz); i: 2.5YR5/8 red (orangebraun); glänzend; gut haftend.

Erhaltung: Rand/Wand/Applike.

Dekor: Efeusträußchen-Applike; Rest des Efeukranzes, der um das Gefäß geschlungen war (horizontal).
Vgl./Dat:: Form: Hübner 1993, Abb. 5-6; MeyerSchlichtmann 1988, 65 Taf. 7. 40 (S 3, 2. Viertel 1. Jh. v. Chr. - Ende 1. Jh. v. Chr./Beginn 1. Jh. n. Chr.); Applike: Hübner 1993, 64-68. 192 Taf. 17, 105; Meyer-Schlichtmann 1988, 213 Taf. 7, 12 (S 3); Kenrick 1985, 60 Abb. 10, 86.2 und S. 63; Bruneau 1970, Taf. 42, D 32; Bruneau 1991, 598 Abb. 1, 41; Warner Slane 1997, 358 Taf. 30, FW 499 (kombiniert mit geritztem Dekor); Pallarés 1986, 95-96 Taf. 7e; Pappa 1994, 87 Abb. 7; Rotroff 1997, 408 Taf. 131, 1648-1649; Zhuravlev 2000, 271 Taf. 138, 3-4. Efeuappliken finden sich auch auf Lampen; vgl. Bruneau 1991, 598 Abb. 2.

KatNr. 10 (Taf. 2, 10; ohne Abb.)

Inv. AG 93/27-1

Befund: römische Agora (M 93/19)

Gattung/Form: Applikenbecher; Hübner Typ I, Form 2-5; Meyer-Schlichtmann S 3

DmR 9,2 cm (?); H 3,4 cm; B 4,2 cm; Ws 0,2-0,3 cm

Ton: 5YR-7/6 reddish yellow (orange); hart; sehr fein, Glimmer (?).

Firnis: schwarz; glänzend; gut haftend.

Erhaltung: Rand/Wand/Applike.

Dekor: Efeusträußchen-Applike; Rest einer hängenden Efeugirlande, die um das Gefäß geschlungen war.

Vgl./Dat.: Form: Hübner 1993, Abb. 5-6; MeyerSchlichtmann 1988, 65 Taf. 7. 40 (S 3, 2. Viertel 1. Jh. v. Chr. - Ende 1. Jh. v. Chr./Beginn 1. Jh. n. Chr.); Applike: Hübner 1993, 64-68. 191-192 Taf. 16-20. 23; MeyerSchlichtmann 1988, 213 Taf. 7, 12 (S 3); Meric 2002, 31 Taf. 5. 91, K 38; Mitsopoulos-Leon 1991, 61 Taf. 72-73, C24-25; Schäfer 1968, 98-99 Taf. 34-35; Bruneau 1991, 607-608 mit Abb. 7; 643, 86; kann auch mit Ritzung kombiniert sein: ebenda 664, 123; s. auch Pallarés 1986, 95-96 Abb. 8f-g und Taf. 7f; Rotroff 1997, 408 Taf. 131, 1647; Zhuravlev 2000, 271 Taf. 138, 5-6.

KatNr. 11 (Abb. 11 Taf. 2, 11)

Inv. AG 90/27-21

Befund: hellenistische Weststoa/Nord 1 - Raubgrube

Gattung/Form: Applikenbecher; Hübner Typ I, Form 2-5; Meyer-Schlichtmann S 3

DmR 11,6 cm; H 6,6 cm; B 8,0 cm; Ws 0,2-0,3 cm

Ton: beige bis graubeige; mittelhart; fein, Glimmer.

Firnis: braun bis rotbraun; eher matt; gut haftend, aber teilweise abgerieben.

Erhaltung: Rand/Wand/Applike.

Dekor: Applike mit großem Platanenblatt (?) und drei Fruchtperlen.

Vgl./Dat.: Hübner 1993, Taf. 23, 127 (Eichenblatt kombiniert mit Efeublatt).

KatNr. 12 (Abb. 12 Taf. 2, 12)

Inv. AG 90/85-1

Befund: hellenistische Weststoa/Süd 2 bzw. 5 - Raubgrube

Gattung/Form: Applikenbecher; Hübner Typ II; MeyerSchlichtmann B 7

DmR ?; H 3,7 cm; B 5,3 cm; Ws 0,3 cm

Ton: 2.5YR6/6 light red (orangerosa); sehr hart; sehr fein, Kalkpseudomorphosen, wenig Glimmer. 
Firnis: a: 2.5 YR5/6 red (rot bis schwarz gefleckt); i: 2.5YR5/8 red; leicht glänzend; gut haftend.

Erhaltung: Wand/Applike.

Dekor: Horizontale Rille auf der Becherwandung, Applike zeigt die Beine und das wallende Gewand einer tanzenden Mänade (?).

Vgl./Dat.: Form: Hübner 1993, Abb. 10-11; MeyerSchlichtmann 1988, 79-80 Taf. 30, 66 (B 7, Datierung nicht eindeutig festgelegt, die Rille könnte ein Hinweis auf die Mitte des 1. Jhs. v. Chr. sein); Applike: Hübner 1993, 203-204 Abb. 39, 262 Taf. 55-58, 260-271, bes. passend Nr. 260; Schäfer 1968, Taf. 22, E 1; Tanzende vgl. Bruneau 1970, Taf. 42, D 35; Bruneau 1991, 614 616, 7-12; Zhuravlev 2000, 271 Taf. 136, 3; 137, 3; zu Mänaden s. Hübner 1993, 149.

\section{Keramik mit Dekor im Westabhang-Nachfolgestil}

Anschließend an die applikenverzierten Becher werden drei Gefäße ganz ähnlicher Form, aber mit differierendem Dekor vorgestellt (KatNr. 13-15 Abb. 13. 14) ${ }^{37}$. Alle drei können als Form S 3 der 'pergamenischen Sigillata' angesprochen werden, die beiden letzten stellen dabei etwas größere Varianten dar. Die Qualität des Scherbens kann als ephesisch identifiziert werden, der Firnis ist meist (braun)orange bzw. rot und kann auch leicht fleckig ausgeprägt sein. Die Oberfläche ist als sigillataartig anzusprechen und glänzt mitunter dementsprechend. Als interessant erweist sich ihr Dekor. Dieser besteht aus Ritzung in Kombination mit Deckweiß, meist in Form von Punkten aufgebracht. KatNr. 13 zeigt dabei geritzte, stehende Achterschleifen, KatNr. 14 einen liegenden Blätterkranz und KatNr. 15 liegende Blütenranken. Die Dekorzonen werden dabei zusätzlich von horizontalen weißen Punktreihen gerahmt. Für die Dekormotive konnten bisher keine direkten Entsprechungen ausfindig gemacht werden - das gilt auch für den Dekor von stehenden Dreiecken mit weißen Punktfüllungen der KatNr. 16 (Abb. 15). Damit ist der Vorschlag, auch diese Gefäße als lokal bzw. regional ephesische anzusehen, auch durch die Dekormotive untermauert.

Diese Art der Gefäßgestaltung auf sigillataähnlichen Gefäßen wurde bisher fast ausschließlich aus Pergamon publiziert. D. Behr definierte den Dekor auf Keramik, welcher nicht nur im Stil der sog. Westabhangkeramik gestaltet, sondern zusätzlich mit Ritzung kombiniert war, als sog. Westabhang-Nachfolgestil. Von den Farben blieb nur das Deckweiß übrig. Als wichtige weitere Kriterien nannte sie sowohl den Übergang von glänzend rotbraunem zu rotem Überzug als auch die Gefäßprofile mit Affinitäten zu den Sigillataformen. Auch rechnete sie diese Spätphase der Westabhangkeramik bereits zu den Vorläufern der pergamenischen Sigillata ${ }^{38}$. Als Gefäßformen mit diesem Dekorstil finden sich bei ihr Skyphoi, Tassen, Schüsseln sowie eine Pyxis. Dieses Repertoire kann um weitere Formen, wie z. B. Thymiateria ${ }^{39}$ oder Näpfe $^{40}$ ergänzt werden. KatNr. 16 belegt, daß auch der Formtyp S 2 bzw. lokale Imitationen dieses Typs damit verziert waren ${ }^{41}$. Der Dekor kann neben vegetabilen und ornamentalen Motiven auch figürliche umfassen $^{42}$. Nicht außer acht gelassen werden darf, daß diese Art des Dekors des öfteren mit

\footnotetext{
37 Vergleichbar den pergamenischen Applikenbechern mit Rotellenhenkeln.

38 Behr 1988, 99. 127-128. 134-137 Abb. 9, 32; 10, 33-36 mit Skyphoi; 140-143 Abb. 11, 37-46; 12, 47-48 sog. Tassen; 153-154 Abb. 15, 64-65 Becher; 159-167 Abb. 17, 74-75; 18, 76-81; 19, 82-85; 20, 86-90 sog. flache Schüsseln; 166-169 Abb. 20, 91; 21, 92-94; 174-175 Abb. 23, 102-103 Deckel; 176 Abb. 25, 111 Pyxis.

39 Radt 2000, 124 Abb. 2.

40 Meyer-Schlichtmann 1988, 190 (N 36).

${ }^{41}$ Entspricht in der Form den sog. koisch-knidischen Schalen, s. u. Kap. 3. Vgl. auch Meriç 2002, 32 Taf. 3, 39; ebenda auch S. 27. 29. 40. 42 Taf. 12, K 126-127 (grautonig, schwarzglänzend gefirnißt, mit ähnlichem Dekor, als sicher lokal ephesisch ausgewiesen, aus Brunnenfüllung B 1, 1-25 n. Chr.). In Pergamon werden sie als flache Schüsseln bezeichnet. Vgl. Behr 1988, 160-167 KatNr. 74-90; ebenso verwendet Mitsopoulos-Leon 1991, 43 Taf. 62, B 140 diese Bezeichnung für ein solches Beispiel aus Ephesos und vergleicht dieses mit entsprechenden Formen in ESB-Qualität.

42 Radt 2000, 125. Er erwähnt Ranken, Blätter, Wellen, Zungenmuster, Perlstäbe und Eierstäbe; ebenda Abb. 2 mit der Darstellung eines Vogels auf Blätter/Blütenranken; Meyer-Schlichtmann 1988, Taf. 37, VK 41 mit der Darstellung eines Greifen, s. dazu Behr 1988, 127-129. Vgl. auch Schäfer 1968, 57. 60 Taf. 11, D 29-30. Aus Delos kennen wir z. B. die Darstellung einer Rosette, s. Bruneau 1970, Taf. 44, D 79.
} 


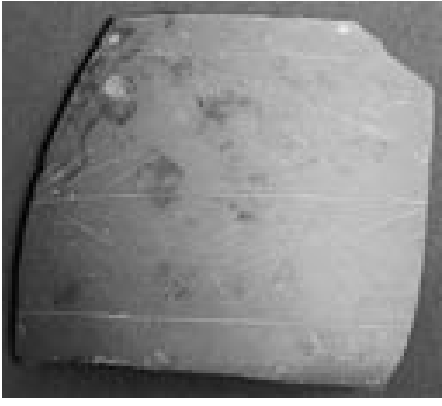

13 KatNr. 14, Inv. AG 90/21-27

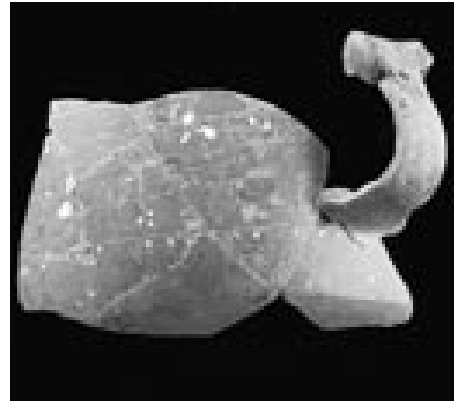

14 KatNr. 15, Inv. AG 96/47-31

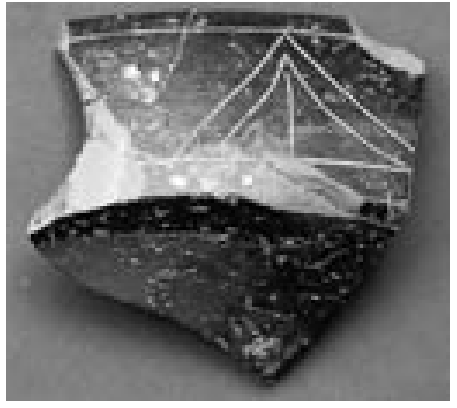

15 KatNr. 16, Inv. AG 96/47-27

Appliken, wie wir sie oben behandelt haben, am selben Gefäß kombiniert wird ${ }^{43}$. Diese Tatsache, die Gefäßformen (S 3), der Überzug (sigillataartig changierend von rotbraun bis rot und besonders braunorange) sowie die relativ großen Fragmente innerhalb der Raubgruben sind wichtige Kriterien für eine nähere zeitliche Einordnung. Fundkomplexe aus Pergamon, Delos und weitere aus Ephesos selbst geben ausreichende chronologische Indizien für die Datierung. Für Pergamon ergeben sich Ansätze für die Zeit vom zweiten Viertel des 1. Jahrhunderts v. Chr. bis zur Zeitenwende ${ }^{44}$. Dabei werden neben den zunehmend schärfer werdenden Formen auch die Firnisfarbe und ihr Wechsel von schwarzbräunlichem über braunorangem zu rotem Firnis als Datierungskriterium miteinbezogen ${ }^{45}$.

Bezüglich Delos sei auf die oben erwähnten Zerstörungshorizonte und die entsprechenden Fundkomplexe verwiesen. In den angesprochenen Befunden sind auch Skyphoi unseres Typs und Dekors enthalten ${ }^{46}$. Beispiele ließen sich auch in Athen, Berenice oder Tel Anafa ausfindig machen $^{47}$.

Aus Ephesos liegen publizierte Exemplare vom Staatsmarkt vor, einerseits aus der Basilika ${ }^{48}$, andererseits aus den bereits erwähnten Brunnenfüllungen A und B $1^{49}$. Die Befunde aus den Grabungen auf der Tetragonos-Agora wurden ebenfalls schon behandelt ${ }^{50}$. Interessant ist die Tatsache, daß KatNr. 14-16 im Gegensatz zu den oben vorgestellten Appliken in relativ großen Fragmenten in den sog. Raubgruben erhalten sind. Dies läßt sicherlich den Schluß zu, daß Gefäße mit sigillataartigem Überzug und Dekor im Westabhang-Nachfolgestil in Ephesos im zweiten und dritten Viertel des 1. Jahrhunderts v. Chr. in Verwendung waren - vielleicht sogar bis zur Zeitenwende ${ }^{51}$.

43 Vgl. Hübner 1993, 35 Taf. 33, 158; Taf. 20, 110; Radt 2000, 127; Schäfer 1968, Abb. 5, 4 Taf. 38, E 99; 100 Taf. 38, E 99-103; Bruneau 1991, 602-603; Warner Slane 1997, 356-359 Taf. 30, FW 499-502.

${ }_{44}$ Meyer-Schlichtmann 1988, 190. Er gibt auch den Hinweis, daß bei Form S 2 ab der Mitte des 1. Jhs. v. Chr. eine gespanntere Bauchkurve zu beobachten ist, ebenda 64.

45 Vgl. Radt 2000, 126-127 mit einer Datierung für das Thymiaterion in die Mitte des 1. Jhs. v. Chr. (bzw. etwas später, dafür spräche die sigillataartig rote Firnisfarbe).

${ }^{46}$ Chatzidakis 2000, 120 Abb. 3, 31 sowie Taf. 63b-c (Unguentarium); Zapheiropoulou - Chatzidakis 1994, 244 Taf. 195, B 14975; Bruneau 1970, D 73. D 79. D 87. D 89. Vgl. auch Pappa 2000, Taf. 55, b1 für Beispiele auf Amorgos.

47 Rotroff 1997, 408 Abb. 98, 1650 bzw. Taf. 131, 1650 (Kontext 200 - spätes 1. Jh. v. Chr.); Kenrick 1985, 62-63 Abb. 10, 85; Warner Slane 1997, 359, FW 504-505 (Becher mit gerader Wandung, ohne Abb., aus Stratum Rom 1B, Beginn 1. Jh. n. Chr.).

48 Mitsopoulos-Leon 1991, 43 Taf. 62, 139-140; 54 Taf. 63, 145-148.

49 Meriç 2002, 32 Taf. 3, K 39-41 (A, späthellenistisch/augusteisch); 40. 42 Taf. 12, K 126-127 (Altstücke, B 1, 1. Viertel 1. Jh. n. Chr.). Auch undekorierte pergamenische Sigillatabecher aus Füllung A, vgl. ebenda 26-27 Taf. 2, 22-23. Vgl. hier Anm. 33.

50 Vgl. Anm. 3 zu den sog. Raubgruben bzw. frühkaiserzeitlichen Aufschüttungen; zu den Südtor-Grabungen s. Gassner 1997, 49. 51 Taf. 8, 129 eine Pyxis im Westabhang-Nachfolgestil (?); ebenda 233-234 Taf. 71, H 11-13 (ohne Dekor, die Beschreibung von Ton und Firnis entspricht unseren Stücken).

51 Eine Bestätigung dieser späten Datierung liefern die Grabungen von S. Ladstätter 1998 im Hanghaus 2, Raum 38 (in Publikationsvorbereitung). Ich danke ihr für diesen Hinweis. 
KatNr. 13 (Taf. 2, 13; ohne Abb.) Inv. AG 95/290-1

Befund: römische Weststoa oder hellenistische Weststoa/ Nord (?) (M 95/59-60) - 'Sentiusschutt'

Gattung/Form: Sigillatabecher; Meyer-Schlichtmann S 3 (mit bereits dreieckig gebildeter Innenlippe)

DmR 11,6 cm; H 4,0 cm; B 5,4 cm; Ws 0,2-0,3 cm Ton: 2.5 YR5/8 red (orange); hart; sehr fein; etwas Glimmer.

Firnis: a: 10R4/6 red bis 2.5YR4/4 dusky red (fleckig rot); i: 2.5YR6/8 red (orange); eher matt; haftet gut. Erhaltung: Rand/Wand.

Dekor: Randbordüre bestehend aus zwei horizontalen geritzten Linien mit weißen Punkten dazwischen, darunter geritzte 8er-Spirale mit weißen Punkten in den Schlingen.

Vgl./Dat.: Form: Meyer-Schlichtmann 1988, 65 Taf. 7. 40 (S 3, 2. Viertel 1. Jh. v. Chr. - Ende 1. Jh. v. Chr./ Beginn 1. Jh. n. Chr.); Dekor: keine direkten Entsprechungen bekannt; Vergleichsstücke aus Ephesos, Pergamon etc. s. Anm. 38-51.

KatNr. 14 (Abb. 13 Taf. 2, 14)

Inv. AG 90/21-27

Befund: hellenistische Weststoa/Nord 1 - Raubgrube Gattung/Form: Sigillatabecher; Meyer-Schlichtmann S 3 (S 1 spät, B 4)

DmR 15,6 cm; H 9,0 cm; B 10,0 cm; Ws 0,3-0,55 cm Ton: braunorange; hart; fein, porös, mitunter gröbere weiße Beimischungen, Glimmer.

Firnis: braunorange; eher matt; gut haftend.

Erhaltung: Rand/Wand.

Dekor: Geritzter Blätterkranz mit weißen Dreipunktblüten in der Hauptzone, diese werden oben und unten von je einer geritzten Linie und einer weißen Punktreihe gerahmt.

Vgl./Dat.: Form: Meyer-Schlichtmann 1988, 65 Taf. 7. 40 (S 3, 2. Viertel 1. Jh. v. Chr. - Ende 1. Jh. v. Chr./Beginn 1. Jh. n. Chr.); ähnlich auch zu Form S 1 spät, ebenda Taf. 7, 3 bzw. B 4; Taf. 9, 62; Dekor: s. KatNr. 13.

KatNr. 15 (Abb. 14 Taf. 2, 15)

Inv. AG 96/47-31

Befund: hellenistische Weststoa/Nord 1 - Raubgrube
Gattung/Form: Sigillatabecher; Meyer-Schlichtmann S 3 (S 1 spät, B 4)

DmR ca. $16,8 \mathrm{~cm}$; $\mathrm{H} 10,4$ und $8,0 \mathrm{~cm}$; B 14,5 und 12,4 $\mathrm{cm}$; Ws $0,4-0,5 \mathrm{~cm}$

Ton: rotbraun; hart; feine weiße Beimischungen, Glimmer.

Firnis: rot; glänzend; gut haftend.

Erhaltung: fünf größere Fragmente erhalten, die zwei größten sind oben angegeben; Rand/Wand, Rotellenhenkel.

Dekor: Geritzte geschwungene Rankenarme mit weißen Dreipunktblüten in der Hauptzone, diese werden oben und unten von je einer geritzten Linie und einer weißen Punktreihe gerahmt. Sechs horizontale Drehrillen bereichern dieses Dekormotiv zusätzlich.

Vgl./Dat:: Meyer-Schlichtmann 1988, 65 Taf. 7. 40 (S 3, 2. Viertel 1. Jh. v. Chr. - Ende 1. Jh. v. Chr./Beginn 1. Jh. n. Chr.); Dekor: s. KatNr. 13.

KatNr. 16 (Abb. 15 Taf. 3, 16)

Inv. AG 96/47-27

Befund: hellenistische Weststoa/Nord 1 - Raubgrube

Gattung/Form: Sigillataschale; Meyer-Schlichtmann S 2; sog. knidische Schale

DmR 18,4 cm; H 5,1 cm; B 7,0 cm; Ws 0,3-0,35 cm

Ton: rotgrau; hart; sehr fein; Glimmer.

Firnis: abwechselnd rot, schwarz bzw. auch braun gefleckt; leicht glänzend.

Erhaltung: Rand/Wand.

Dekor: Gemalter und geritzter Dekor sind kombiniert: Zwischen je zwei geritzten horizontalen Linien finden sich ebenfalls geritzte stehende Dreiecksmotive, welche mit weißen Firnispunkten zusätzlich gegliedert sind; die Dreiecke werden durch weitere weiße Dreipunktmotive voneinander getrennt. Diese Randbordüre wird mittels einer horizontalen Reihe weißer Punkte nach unten hin abgeschlossen. Die aufgemalten weißen Punkte sind oft stark abgerieben. Zusätzlich wechselt der Firnisuntergrund von schwarz auf rot.

Vgl./Dat.: Form: Meyer-Schlichtmann 1988, 64-65 Taf. 7. 29 (S 2, Ende 2. Jh./Beginn 1. Jh. v. Chr. - Beginn 1. Jh. n. Chr.); knidische Schalen s. u. Kap. 3; Dekor: ähnlich Meriç 2002, 32 Taf. 3, 39; 40. 42, Taf. 12, K 126-127; Mitsopoulos-Leon 1991, 54 Taf. 62, B 140; s. KatNr. 13.

\section{Knidische Schalen mit Dekor}

KatNr. 16 (Abb. 15) bildet den Übergang zur letzten Gruppe. Zählt das Stück vom Dekor her zur vorigen Gruppe, so ist seine Form als lokale Nachahmung der bekannten knidischen Schalen anzusprechen. Bei den sog. knidischen bzw. auch koisch-knidischen Schalen ${ }^{52}$ handelt es sich um weitausladende Trinkschalen auf niedrigem Fuß. Sie besitzen einen senkrecht abknickenden, hohen Rand, an welchem seitlich zwei П-förmige Henkel angebracht sind. Die Gefäßform dürfte in Knidos selbst als Weinschale um ca. 200 v. Chr. eingeführt und bis in die mittlere Kaiserzeit

\footnotetext{
52 Von J. W. Hayes mit Kos und von P. M. Kenrick schließlich mit Knidos in Verbindung gebracht. Vgl. Hayes 1971,
} 258 Nr. 8 und Kenrick 1985, 58. 


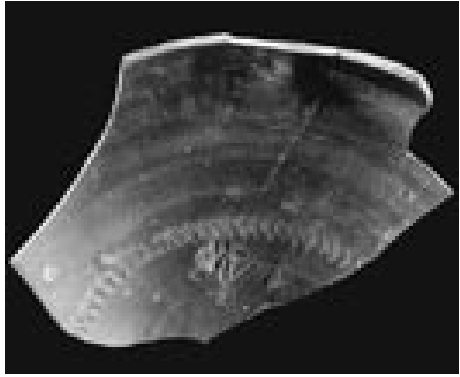

16 KatNr. 17, Import aus Knidos, Inv. AG 96/47-28

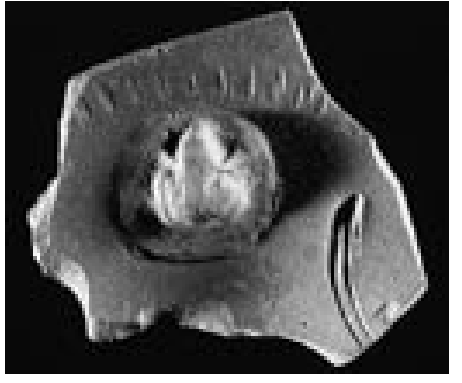

17 KatNr. 18, Import aus Knidos, Inv. AG 98/104-1

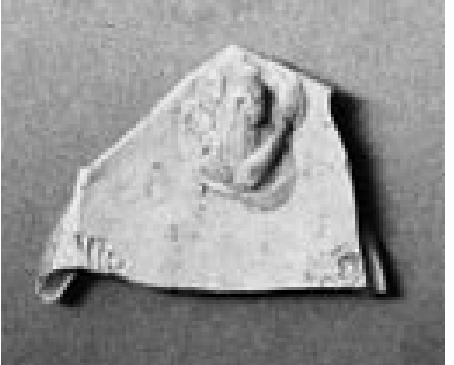

18 KatNr. 19, Import aus Knidos (?), Inv. AG 90/27-22

produziert worden $\operatorname{sein}^{53}$. Besonders charakteristisch und bekannt ist neben der Form ein für knidische Keramik typisches graugebranntes Fabrikat ${ }^{54}$. Für das zweite Viertel des 2. Jahrhunderts v. Chr. konnten weithin erfolgte Exporte ${ }^{55}$, welche einzelne lokale Keramikproduktionen beeinflußten, festgestellt werden ${ }^{56}$. In der Zeit des späten Hellenismus sind sie innen oft mit Appliken ${ }^{57}$ und/oder mit Stempel mit vegetabilen oder figürlichen Motiven und/oder Roulettierungen dekoriert. Beliebte Stempelmotive sind dabei Palmetten, Weinranken, Lotusblüten, Liliensträußchen etc.

Die zwei folgenden Beispiele aus Ephesos, KatNr. 17-18 (Abb. 16. 17), konnten als knidische Importe identifiziert werden. Scherben und Firnis ${ }^{58}$, aber auch Profil und aufgebrachter Dekor stimmen mit den aus Knidos bekannten Produkten überein. Bei KatNr. 17 ist im Inneren innerhalb einer Roulettierung ein Liliensträußchen eingestempelt ${ }^{59}$. Bei KatNr. 18 sind innerhalb einer etwas einfacher gestalteten Roulettierung ein applizierter Frosch $^{60}$ und die Hälfte eines gestempelten, halbrunden Blattes zu erkennen. Obwohl sich nur dieser kleine Ausschnitt erhalten hat, wird klar, daß sich ursprünglich die Darstellung eines See- bzw. Teichufers im Inneren dieser Trinkschale befand. Beim Leeren des Gefäßes erschienen nach und nach die dargestellten Teile, wohl beginnend mit den zuoberstliegenden Augen des Frosches.

Auch das Fragment KatNr. 19 (Abb. 18), das den Abschluß bildet, vereint als Schmuck im Inneren applizierten und gestempelten Dekor ${ }^{61}$; neben einer Froschapplike sind zwei nicht näher zu benennende, kleine Stempelmotive zu erkennen. Auch dieses Stück könnte aufgrund seiner

53 Kenrick 1985, 58; Kögler 1996, 64. Ladstätter u. a. 2003, 31 konnte für Ephesos eine Laufzeit dieser Form bis in flavische Zeit nachweisen, s. ebenda auch Anm. 126.

${ }^{54}$ Kenrick 1985, 58. Der Scherben ist sehr hart, im Kern meist grau gebrannt, die Oberfläche kann dazu orange sein. Daneben sind wenig Glimmer und meist zahlreiche weiße Einschlüsse zu beobachten. Sie sind innen zur Gänze und außen nur teilweise von einem sehr dünnen, meist ebenfalls matten grauen Firnis überzogen. Vgl. Mandel 1996, 61-62 und hier KatNr. 17-18. Daneben finden sich aber auch andere Ton- bzw. Firnisfarben bei Produkten, die in Knidos hergestellt wurden. Mandel 1996, 62 spricht sogar davon, die knidische Keramik in sog. bunte und graue Fabrikate einteilen zu müssen. Ein vielfarbiger, bunter Eindruck scheint von den Töpfern beabsichtigt gewesen zu sein.

55 Rotroff 1997, 233-234 Taf. 96, 1576-1579 mit Anm. 66-67 zu den Athener Fundgruppen und Fundkontexten in Pergamon, Delos und Korinth. Vgl. auch Warner Slane 1997, 368-369 Taf. 540-541. Zur Verbreitung s. Rotroff 1997, 233-234 mit Anm. 61; Kenrick 1985, 59-61.

56 Meyer-Schlichtmann 1988, 64; Rotroff 1997, 119 Taf. 38-39, 395-404; Ladstätter u. a. 2003, 52 f. KatNr. 89-90; vgl. hier KatNr. 16. Die lokalen Imitationen können dabei aufgrund des Fabrikats, aber auch am spannungsreicheren Profil bzw. dem weniger hohen Rand erkannt werden.

57 Kögler 1996, 64; Bruneau 1991, 657 Nr. 75 (Delos).

58 Sog. Knidian Grey ware, vgl. Rotroff 1997, 233.

59 Weitere Vergleichsbeispiele s. Katalog.

${ }^{60}$ Erinnert werden kann hier an KatNr. 8, einen Applikenbecher der Form S 8 aus der Mitte des 1. Jhs. v. Chr., welcher im Gefäßinneren ebenfalls eine Froschapplike aufweist.

${ }^{61}$ Auf knidischen Schalen ist applizierter und gestempelter Dekor in Kombination besonders beliebt; persönliche Information P. Kögler, Frankfurt. 


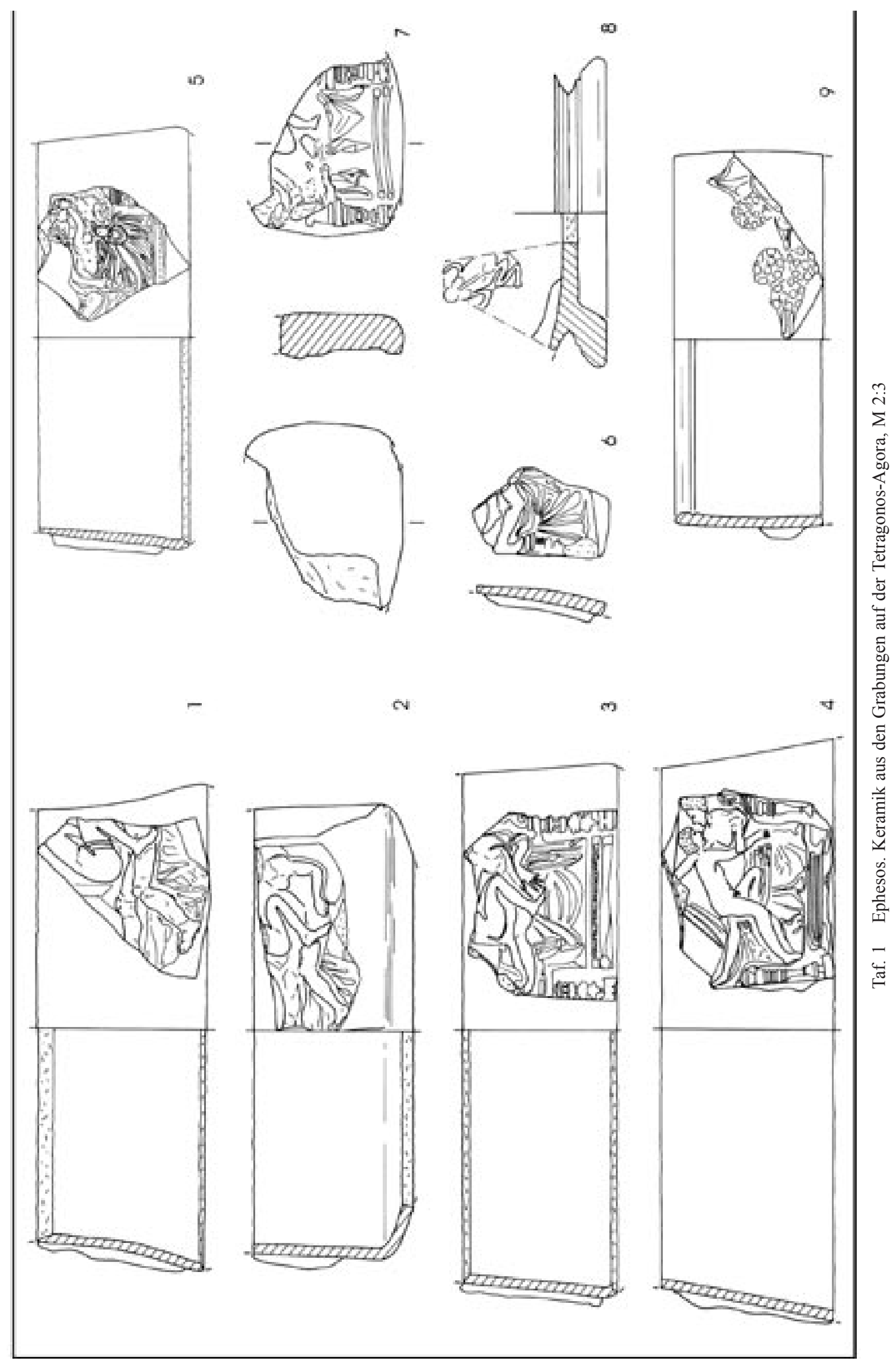


Scherbenqualität und gewisser Ähnlich- bzw. Nachlässigkeiten bei der Gestaltung der Froschappliken $^{62}$ aus Knidos stammen. Einzig die Form des stärker profilierten Fußes bzw. das Gefäßprofil an sich lassen es nicht zu, dieses Bodenfragment als knidische Schale anzusprechen. Es erinnert vielmehr an frühe Sigillataschälchen.

Für eine zeitliche Einordnung der knidischen Schalen lassen sich Fundkontexte, wie großteils bereits oben genannt, aus Athen, Delos, Pergamon und Ephesos selbst anführen ${ }^{63}$. Diese können durch weitere auf Samos, Amorgos, Knossos, Paphos und Kaunos ergänzt werden ${ }^{64}$. Die hier vorgelegten Beispiele KatNr. 16-18 stammen dabei aus den in augusteischer Zeit geschlossenen Raubgruben der Weststoa, KatNr. 19 gehört zu einem zeitgleichen Fundkomplex im Bereich des Nordtores der Tetragonos-Agora ${ }^{65}$. Zusammenfassend bedeutet dies, daß eine Konzentration der Funde im allgemeinen wiederum am Ende des 2. und im 1. Jahrhundert v. Chr. bzw. auch im 1. Jahrhundert n. Chr. auszumachen ist. Dabei dürften die grautonigen knidischen Schalen besonders in der zweiten Hälfte des 1. Jahrhunderts v. Chr. anzutreffen sein. Hier scheint sich die ephesische Evidenz mit den in Athen gemachten Beobachtungen zu decken ${ }^{66}$.

KatNr. 17 (Knidos) (Abb. 16 Taf. 3, 17)

Inv. AG 96/47-28

Befund: hellenistische Weststoa/Nord 1 - Raubgrube

Gattung/Form: knidische Schale

DmR ca. $21 \mathrm{~cm}(?) ; \mathrm{H} 4,8 \mathrm{~cm}$; B 13,8 cm; Ws 0,3-0,5 $\mathrm{cm}$

Ton: grau im Kern, a: rosa bis orange; sehr hart; feinste weiße Beimischungen.

Firnis: graubraun bis rotbraun, a: teils rot, sonst tongrundig; matt; gut haftend.
Erhaltung: Wand.

Dekor: Gestempeltes Liliensträußchen mit Früchten, umgeben von grob ausgeführter Roulettierung.

Vgl./Dat.: Form: Kögler 1996, Taf. 20, 1. 3. 4; Rotroff 1997, Abb. 96, 1576-1578 (kurz vor der Mitte des 2. Jhs. v. Chr - 1. Jh. n. Chr.); Meyer-Schlichtmann 1988, 64-65 Taf. 7. 29 (S 2, Ende 2. Jh./Beginn 1. Jh. v. Chr. - Beginn 1. Jh. n. Chr.); Dekor: 'fleur de lys'/Lilienstempel: vgl. Bruneau 1970, 249 Taf. 43, D 66; R. Tölle-Kastenbein, Das Kastro Tigani, Samos XIV (1974) 162 Abb. 270;

${ }^{62}$ Auffällig ist die unterschiedliche Gestaltung der einzelnen Froschappliken. Ist jene von KatNr. 8 detailreich gestaltet und der Körperform des Frosches entlang ausgeschnitten, so ist jene von KatNr. 18 als kleine runde Scheibe gebildet, auf welcher ein schemenhaft geformter Frosch sitzt. Die Applike von KatNr. 19 schließt sich eher letzterem Beispiel an. Auch sie besitzt eine Auflagefläche, allerdings länglich, sowie einen nachlässig ausgeführten Frosch. Vgl. dazu knidische Importe auf Amorgos, Pappa 1994, 91. 95 mit Abb. 13.

${ }^{63}$ Athen: Rotroff 1997, 119: Imitationen (frühes 2. Jh. v. Chr. bis frührömische Kontexte, besonders häufig in 'deposits' der 1. Hälfte des 1. Jhs. v. Chr.); ebenda 234: 'knidische graue Ware' (kurz vor der Mitte des 2. Jhs. v. Chr. bis weit ins 1. Jh. n. Chr.); Delos: Bruneau 1971, 256 Taf. 47, D 174-175; Zapheiropoulou - Chatzidakis 1994, 243-244 Taf. 1994, B 14994. 14813; Peignard 1997, 314 Taf. 234e; Chatzidakis 2000, Abb. 3, 32 (Ende 2. Jh. 1. Drittel 1. Jh. v. Chr.); Pergamon: Meyer-Schlichtmann 1988, 64-65 zu Form S 2; Ephesos: Ladstätter u. a. 2003 KatNr. 89-91 (Brunnenfüllung 2, ca. 100 v. Chr.); Gassner 1997, 49-50 Taf. 7, 117-119 (Drainage-Füllung beim Südtor, letztes Drittel 2. Jh. v. Chr.); sog. Straße II-Horizont der Grabungen in der Weststoa der Tetragonos-Agora mit zwei knidischen Schalen, orangetonig (in Publikationsvorbereitung, Gruppe H 5, Ende 2. Jh./erste Dekaden 1. Jh. v. Chr.); Meriç 2002, 27. 29 Taf. 3, 29-32 (Füllung B 1, späthellenistisch-augusteisch); ebenda 29 Taf. 3, 33-34 zeitlich später anzusetzende knidische Schalen mit Absatz am Randansatz außen (Füllung B 3, neronisch). - Weitere Kontexte und Fundorte im Mittelmeerraum nennt Rotroff 1997, 23-234 mit Anm. 61. 66-67. Aus Knidos selbst sind noch keine entsprechenden Befunde publiziert. Vgl. U. Mandel in: 5. Kongreß zur hellenistischen Keramik, Chania 1997 (2000) 179 mit Anm. 1 und 3.

${ }^{64}$ Samos: K. Tsakos in: L. Kypraiou (Hrsg.), Hellenistic pottery from the Aegean (1994) 154 Abb. 9 (Füllung 130-125 v. Chr. datiert); Amorgos: Pappa 1994, 93. 95 Abb. 13 (aus Kontexten der Mitte des 2. Jhs. v. Chr. bis zum beginnenden 1. Jh. n. Chr.); Pappa 2000, 114 Taf. 61, b6-7 (letztes Viertel 2. Jh. - Beginn 1. Jh. v. Chr.); Knossos: Hayes

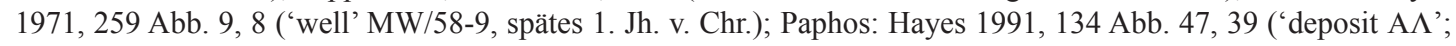
ca. 110-100 v. Chr.?); 168 Abb. 59, 4 ('well' 20, spätes 2. Jh. v. Chr.); 182 Abb. 62, 30 ('well' 18, spätaugusteisch); Kaunos: Schmaltz 1996, 72.

${ }_{65}$ Vgl. ÖJh 68, 1999, Jahresbericht 1998, 14-15 mit Abb. 2 (P. Scherrer). Auch diese Aufschüttung enthält typische ESA-Formen des 1. Jhs. v. Chr., z. B. Hayes 1985, Form 11, protoaugusteisch. Direkt darunter stieß man auf ein frühhellenistisches Niveau. Vgl. ÖJh 70, 2001, Jahresbericht 2000, 257; ÖJh 71, 2002, Jahresbericht 2001, 362.

${ }^{66}$ Rotroff 1997, 233-234 macht darauf aufmerksam, daß in Athen die eigentlichen grauen knidischen Schalen gehäuft in späteren Kontexten (meist 2. Hälfte 1. Jh. v. Chr. und später) als ihre attischen Imitationen auftreten. 


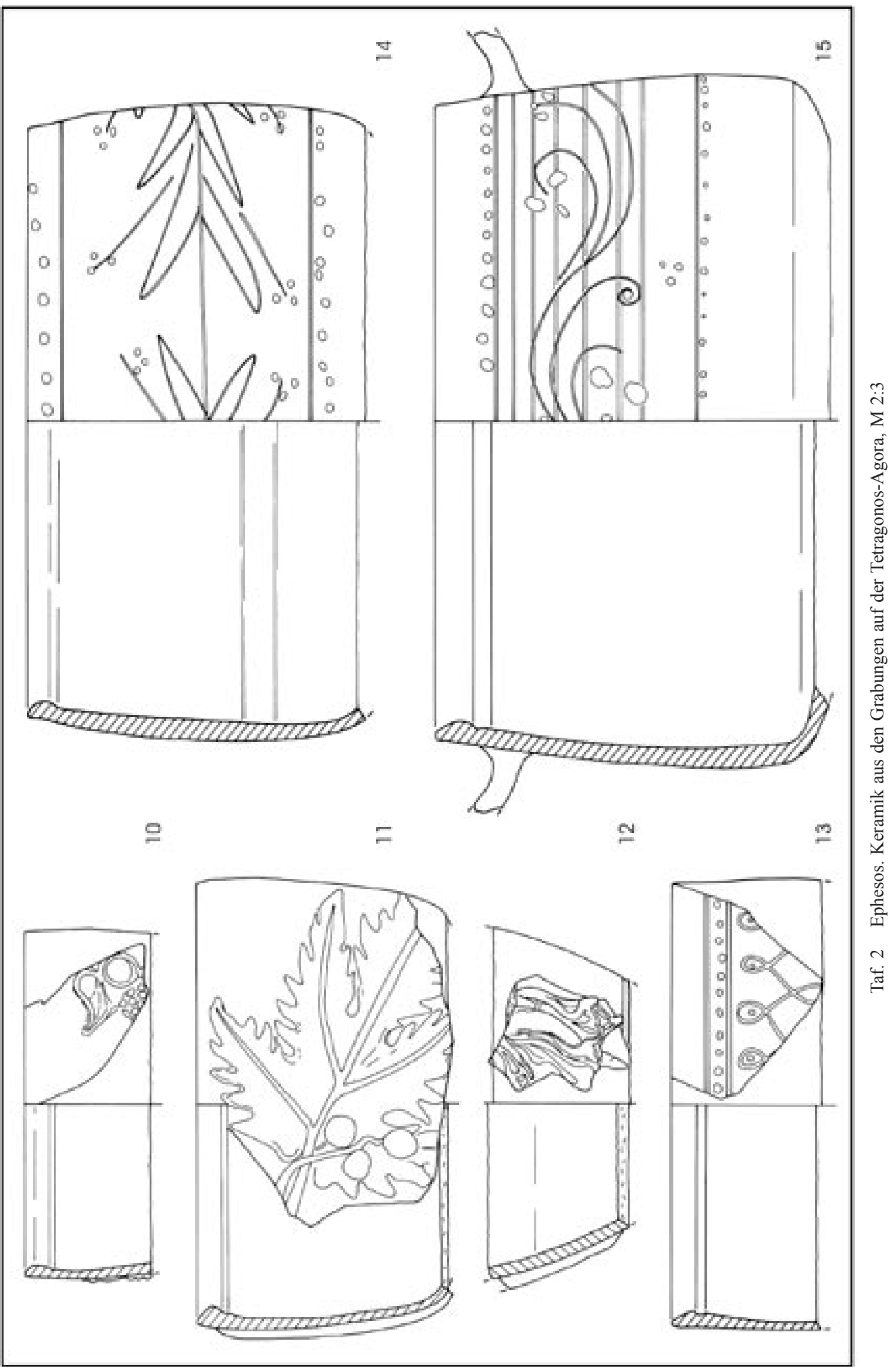


Kenrick 1985, 59-61 Abb. 10, 82.3-4 (mit weiteren Beispielen in Athen und Antiochia); unser Stempel liegt bezüglich der Größe zwischen Kenrick 1985, 65 Taf. 2, $\mathrm{X} 24$ und $\mathrm{X} 25$.

KatNr. 18 (Knidos) (Abb. 17 Taf. 3, 18)

Inv. AG 98/104-1

Befund: römisches Nordtor (M 98/13)

Gattung/Form: knidische Schale

DmB 6,2 cm; H 2,5 cm; B 5,0 cm; Ws 0,3 cm

Ton: 2.5YR-N4 dark gray (grau), an Oberfläche leicht orange; sehr hart; leicht porös, weiße Beimischungen.

Firnis: a: tropfenartige Reste von grauem Firnis, sonst tongrundig; i: 5YR5/1 gray (grau); matt; gut haftend.

Erhaltung: Standring/Boden/Wand.

Dekor: Kreisförmige Froschapplike, relativ schematisch; rechts davon Teil eines gestempelten halbrunden Blattes; beide werden von einer einfachen, grob ausgeführten Roulettierung umgeben; Frosch ca. 1,7 cm lang.

Vgl./Dat.: Form: Kögler 1996, Taf. 20, 1. 3. 4; Rotroff 1997, Abb. 96, 1576-1578 (kurz vor der Mitte des 2. Jhs. v. Chr - 1. Jh. n. Chr.); Meyer-Schlichtmann 1988, 64-65 Taf. 7. 29 (S 2, Ende 2. Jh./Beginn 1. Jh. v. Chr. - Beginn 1. Jh. n. Chr.); Dekor: Kögler 1996, Taf. 20, 4 (Knidos); Schmaltz 1996, 72 (Kaunos); Pappa 1994, 91. 95 mit
Abb. 13 (Frösche, Stempel und Roulettierung; Amorgos); Bruneau 1970, 249 Taf. 43, D 66; Kenrick 1985, 58-61 Abb. 10, 82.1-4 (Berenice) mit Aufzählung weiterer Gefäße mit Froschappliken in Athen und Antiochia; diese können auch auf Lampen appliziert sein, s. Ph. Bruneau, Les lampes, Délos XXVI (1965) 98, 4382.

KatNr. 19 (Knidos?) (Abb. 18 Taf. 3, 19)

Inv. AG 90/27-22

Befund: hellenistische Weststoa/Nord 1 - Raubgrube

Gattung/Form: Schälchen (?)

DmB 5,8 cm; H 2,3 cm; B 5,8 cm; Ws 0,2-0,3 cm

Ton: 2.5YR-N4 dark gray (grau); sehr hart; leicht porös, weiße Beimischungen.

Firnis: i: orangebraun; matt, dünn; gut haftend; a: nur teilweise gefirnißt, sonst tongrundig.

Erhaltung: Standring/Boden/Wand.

Dekor: Längliche Froschapplike, relativ schematisch; rechts davon Reste zweier Stempelmotive erhalten; Frosch ca. 1,75 cm lang.

Vgl./Dat.: Dekor: s. KatNr. 18; bes. H. S. Robinson, Pottery of the Roman period, Agora V (1959) 13-14, F 29-32 Taf. 39 mit P 20456+P 6915 (Froschapplike und ähnliche Stempelmotive).

Diese kleine Auswahl spezifischer Gefäßgattungen läßt nicht nur die starke Ausprägung mancher Zentren mit Keramikindustrie an der kleinasiatischen Westküste erahnen - dazu zählen Städte wie Pergamon, Ephesos oder Knidos nun einmal -, sondern macht auch deutlich, wie groß die Einflußnahme untereinander ist. Sind bestimmte Keramikgattungen und Formen einmal gestaltet und am Markt, werden sie anderenorts abgeformt bzw. schnellstens imitiert. Das können dekorierte pergamenische Sigillatabecher, ephesische (ionische) Reliefbecher oder EphesosLampen, aber auch knidische Schalen sein. Auch die verwendeten Motive können übernommen werden. Nur die Beobachtung kleinster Details bezüglich der Scherbenqualität, des Profils, der Fußgestaltung, der Proportionen, des Motivs und der technischen Ausführung eröffnet einen Weg, Importe von lokalen Imitationen eindeutig zu scheiden. So kann hier für die meisten Applikenbecher und Becher mit Dekor im Westabhang-Nachfolgestil, obwohl sie in absoluter Tradition pergamenischer Sigillataformen zu stehen scheinen, eine lokal bzw. regional ephesische Entstehung konstatiert werden. Der Unterschied liegt im Scherben, vielleicht auch in der Reduktion auf bestimmte Motive (Symplegmata, Efeu) sowie im verwendeten Motiv selbst. Wird der Motivschatz übernommen, kann er sowohl auf verschiedenen Gattungen als auch auf zeitlich späteren Formen angebracht werden - erinnert sei zu ersterem an das Fragment KatNr. 8 mit seiner innen angebrachten Froschapplike, wie man sie sonst nur von knidischen Schalen kennt, bzw. zu letzterem an die kelchartigen Applikenbecher aus Ephesos mit den übernommenen Symplegmata-Darstellungen ${ }^{67}$. War ein Produkt in der Antike also 'erfolgreich', darf man Exporte und Nachahmungsversuche an zahlreichen Orten erwarten ${ }^{68}$. Damals wie heute wurden Erfolgsrezepte exportiert und kopiert, und damals wie heute liegt der Unterschied (ganz besonders) im Detail.

67 Vgl. KatNr. 7 mit Zabehlicky-Scheffenegger - Schneider 2000, 109 Abb. 3, 1 r.

68 Offen bleibt dabei derzeit in vielen Fällen noch die Frage nach dem Entstehen und erstmaligem Auftreten bestimmter Formen und Gattungen. Die chronologischen Sequenzen an den einzelnen Produktionsorten sind noch nicht eindeutig geklärt. 


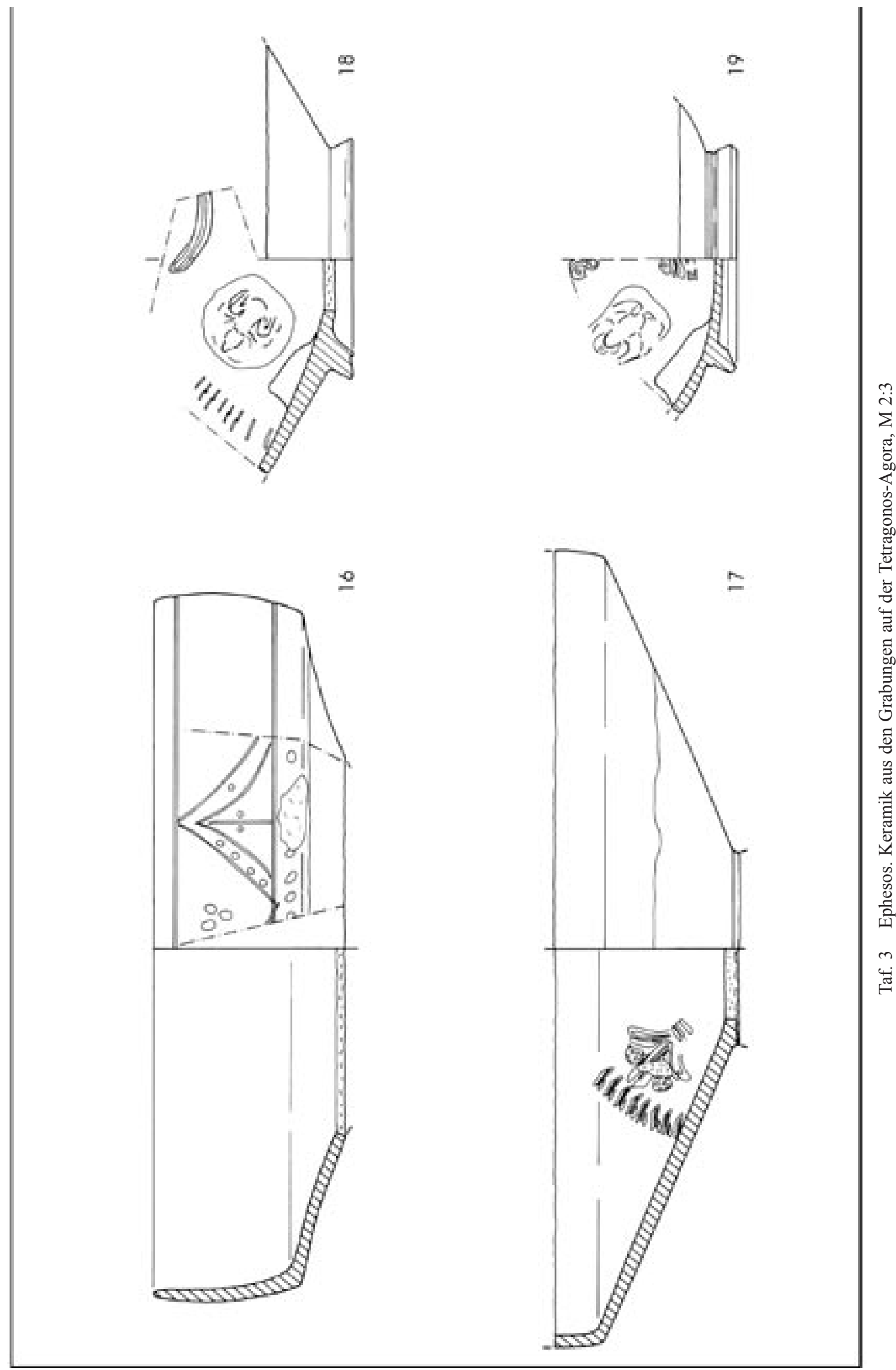


Dabei wurde die Frage nach der zeitlichen Stellung der hier besprochenen Gefäße sowohl in Ephesos als auch außerhalb angeschnitten; speziell zu den applikenverzierten Bechern (s. o. Kap. 1) wurden auch die derzeitigen Kenntnisse zum ursprünglichen Entstehungsort und dem Exportradius angesprochen sowie die Frage nach ihrer Funktion nochmals andiskutiert. Diese Becher sollten vielleicht einfach nur als Trinkbecher innerhalb des Tafelservices betrachtet werden. Sie als Trinkbecher, welche beim 'Symposion' Verwendung fanden, anzusprechen ${ }^{69}$, erscheint aufgrund gewisser Hinweise äußerst verlockend, kann aber noch nicht eindeutig bewiesen werden. Als Argumente dafür könnten neben den zur Anwendung gelangten dionysischen Motiven hier besonders die aus Delos vorgestellten applikenverzierten Gefäße in ihrer Gesamtheit dienen $^{70}$. Dabei handelt es sich um Trink-, Gieß- oder Mischgefäße. Diese sind vergleichbar mit jenen uns bekannten Symposions-Gefäßformen, welche aus mit Einzelstempeln dekorierten Modeln gewonnen wurden; das sind (meist) halbkugelige Reliefbecher verschiedener Größen, Reliefkratere, -kannen, -amphoren oder auch -tassen ${ }^{71}$. Die Hauptproduktionszeit der applikenverzierten Becher würde auch nach dem Auslaufen der Massenproduktion von Reliefbechern einsetzen und könnte somit als langsame Ablöse bzw. schließlich Nachfolge dieser angesehen werden. Auffällig dabei ist die Tatsache, daß die Anzahl der applikenverzierten Gefäße in Pergamon deutlich höher zu sein scheint als in Ephesos. Ungeachtet des unterschiedlichen Forschungs- und Publikationsstandes und eingedenk der daraus resultierenden Unsicherheit, Schlüsse zu ziehen, könnte eine Erklärung dieses Umstandes in der stärker nach Rom orientierten Ausprägung der Metropole Ephesos gefunden werden. In Ephesos scheint die Bevölkerung, die sicherlich einen sehr hohen Anteil italischstämmiger Bürger aufwies, im Laufe des 1. Jahrhunderts v. Chr. immer weniger in den griechisch-hellenistischen Traditionen (wie es z. B. das Abhalten von Symposien andeuten würde) verhaftet zu sein - sicherlich weniger als jene von Pergamon oder jene in den Küstengebieten der Schwarzmeerregion. Allein dadurch bestanden bereits eine geringere Nachfrage und weniger Bedarf an diesem Satz von Geschirr in Ephesos selbst $^{72}$; daß die applikenverzierten Becher aber dort hergestellt und exportiert wurden, belegen die angeführten Beispiele und Vergleiche ${ }^{73}$.

69 Bruneau 1991, 606-609; Z. Kotitsa, Hellenistische Keramik im Martin von Wagner Museum der Universität Würzburg (1998) 141.

70 Derzeit bekannte Motive aus Ephesos: Efeuranken bzw. -girlanden, erotische Symplegmata, Mänaden. Derzeit bekannte Motive und Formen aus Delos: Kratere, Becher verschiedenen Typs, Rhyta, Filterkrüge, Kannen; Figuren des dionysischen Thiasos' (Dionysos, Mänade, Tänzerinnen, Satyr, Silen, Eros, Kitharöde), erotische Symplegmata, groteske oder ithyphallische Figuren, Masken, Eroten, Efeuranken, -girlanden (auch von Eroten getragen); vgl. Bruneau 1991, 600-609.

71 A. Laumonier, La céramique hellénistique à reliefs. Ateliers «Ioniens», Délos XXXI (1977) Taf. 131-134.

72 Delos, der Handels- und Umschlagplatz für Waren aller Art im beginnenden 1. Jh. v. Chr., kann hier wohl aufgrund seines ebenfalls hohen römischen Bevölkerungsanteiles am ehesten mit Ephesos verglichen werden. Die Masse der Funde an hellenistischen Reliefbechern überwiegt bei weitem den eher geringen Anteil an applikenverzierter Keramik des 1. Jhs. v. Chr. Vgl. die relevanten Publikationen von Laumonier (Anm. 71) mit ca. 10000 Stücken und Bruneau 1991 mit ca. 150 Beispielen. Pergamon scheint hier andere Anteiligkeiten aufzuweisen.

73 s. dazu auch die Beispiele aus der Sammlung Candolle in Genf, welche in Smyrna entstanden ist. Vgl. CVA Genève (1) III N Taf. 31, bes. 11-28 (Model bzw. Becherfragmente mit Efeu- oder erotischem Symplegma-Motiv). 


\section{Abkürzungen und abgekürzt zitierte Literatur}

$\begin{array}{llll}\text { a } & \text { außen } & \text { ESB } & \text { Eastern Sigillata B } \\ \text { AG } & \text { Tetragonos-Agora } & \mathrm{i} & \text { innen } \\ \text { DmB } & \text { Durchmesser/Boden } & \mathrm{M} & \text { Münze } \\ \text { DmR } & \text { Durchmesser/Rand } & \text { Ws } & \text { Wandstärke }\end{array}$

Behr 1988

Bruneau 1970

Bruneau 1991

Chatzidakis 2000

Gassner 1997

Hayes 1971

Hayes 1985

Hayes 1991

Hübner 1993

Kenrick 1985

Kögler 1996

Ladstätter 2000

Ladstätter u. a. 2003

Ladstätter - Lang-Auinger 2001

Mandel 1996

Meriç 2002

Meyer-Schlichtmann 1988

Mitsopoulos-Leon 1991

Pallarés 1986

Pappa 1994

Pappa 2000

Peignard 1997

Radt 2000

Rotroff 1996

Rotroff 1997

Schäfer 1968

Schmaltz 1996
D. Behr, Neue Ergebnisse zur pergamenischen Westabhangkeramik, IstMitt 38, 1988, 97-178.

Ph. Bruneau (Hrsg.), L'îlot de la Maison des Comédiens, Délos XXVII (1970).

$\mathrm{Ph}$. Bruneau, La céramique pergaménienne à reliefs appliqués de Délos, $\mathrm{BCH} 115$, 1991, 597-666.

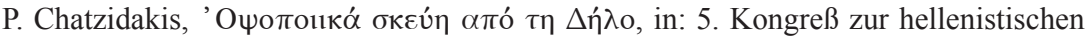
Keramik, Chania 1997 (2000) 115-130.

V. Gassner, Das Südtor der Tetragonos-Agora. Keramik und Kleinfunde, FiE XIII 1, 1 (1997).

J. W. Hayes, Four early Roman groups from Knossos, BSA 66, 1971, 249-275.

J. W. Hayes, Sigillate Orientale, in: EAA II (1985) 9-96.

J. W. Hayes, The Hellenistic and Roman pottery. Paphos III (1991).

G. Hübner, Die Applikenkeramik von Pergamon. Eine Bildersprache im Dienst des Herrscherkultes, PF 7 (1993).

P. M. Kenrick, Excavations at Sidi Khrebish. Benghazi (Berenice) III 1: The fine pottery, 5. Suppl. LibyaAnt. (1985).

P. Kögler, Trinkschalen mit П-förmigen Henkeln, in: Hellenistische und kaiserzeitliche Keramik des östlichen Mittelmeergebietes, Kolloquium Frankfurt 24.-25. April 1995 (1996) 64

S. Ladstätter, Ein flavischer Fundkomplex aus dem Hanghaus 2 von Ephesos, Rei Cretariae Romanae Fautorum Acta 36, 2000, 97-103.

S. Ladstätter - Ch. Rogl - A. Giuliani - T. Bezeczky - B. Czurda, Ein hellenistischer Brunnen in SR 9c, in: C. Lang-Auinger (Hrsg.), Hanghaus 1 in Ephesos. Funde und Ausstattung, FiE VIII 4 (2003) 22-85.

S. Ladstätter - C. Lang-Auinger, Zur Datierung und kunsthistorischen Einordnung einer Apollon-Kitharodos-Statuette, in: F. Krinzinger (Hrsg.), Studien zur hellenistischen Keramik in Ephesos, 2. ErghÖJh (2001) 71-81.

U. Mandel, Keramik aus Knidos - ein Vorbericht, in: Hellenistische und kaiserzeitliche Keramik des östlichen Mittelmeergebietes, Kolloquium Frankfurt 24.-25. April 1995 (1996) 61-63.

R. Meriç, Späthellenistisch-römische Keramik und Kleinfunde aus einem Schachtbrunnen am Staatsmarkt in Ephesos, FiE IX 3 (2002).

C. Meyer-Schlichtmann, Die pergamenische Sigillata aus der Stadtgrabung von Pergamon, PF 6 (1988).

V. Mitsopoulos-Leon, Die Basilika am Staatsmarkt in Ephesos. Kleinfunde. 1. Teil: Keramik hellenistischer und römischer Zeit, FiE IX 2, 2 (1991).

F. Pallarés. Il relitto della nave Romana di Spargi, Archeologia Subacquea 3, 37./38. Suppl. BdA (1986) 89-102.

V. Pappa, From the Hellenistic pottery of Minoa, in: L. Kypraiou (Hrsg.), Hellenistic pottery from the Aegean (1994) 81-99.

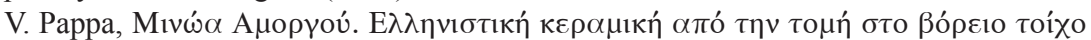

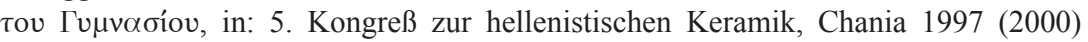
$105-114$.

A. Peignard, La vaisselle de la Maison des Sceaux, Délos, in: 4. Kongreß zur hellenistischen Keramik, Mytilene 1994 (1997) 308-316.

W. Radt, Ein sigillata-rotes Thymiaterion mit Ritzdekor im Westabhang-Nachfolgestil aus Pergamon, Rei Cretariae Romanae Fautorum Acta 36, 2000, 123-128.

S. I. Rotroff, Gnomon 68, 1996, 356-361 (zu Hübner 1993).

S. I. Rotroff, Hellenistic pottery. Athenian and imported wheelmade table ware and related material, Agora XXIX (1997).

J. Schäfer, Hellenistische Keramik aus Pergamon, PF 2 (1968).

B. Schmaltz, Hellenistische und kaiserzeitliche Keramik aus Kaunos, in: Hellenistische 

24.-25. April 1995 (1996) 71-73.

Schwarzer 1999 H. Schwarzer, Die Applikenware von Pergamon - Ein Kultgeschirr des Herrscherkultvereines der Attalisten?, IstMitt 49, 1999, 298-300.

Warner Slane 1997 K. Warner Slane, The fine Wares, in: S. C. Herbert (Hrsg.), Tel Anafa II 1. The Hellenistic and Roman pottery, 10. Suppl. JRA (1997) 249-393.

Zabehlicky-Scheffenegger Schneider 2000

S. Zabehlicky-Scheffenegger - G. Schneider, Applikenverzierte Gefäße aus Ephesos, Rei Cretariae Romanae Fautorum Acta 36, 2000, 105-112.

Zapheiropoulou -

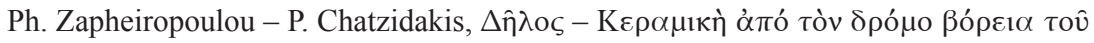

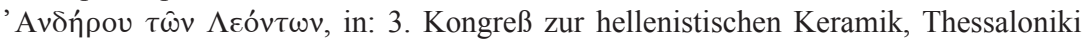
1991 (1994) 235-248.

Zhuravlev 2000

D. Zhuravlev, A skyphos from the House of Chrysaliskos and Pergamene pottery import in the Bosporan Kingdom, in: 5. Kongreß zur hellenistischen Keramik, Chania 1997 (2000) 269-272.

Dr. Christine Rogl

Institut für Kulturgeschichte der Antike, Österreichische Akademie der Wissenschaften, Bäckerstraße 13, A-1010 Wien

E-Mail: christine.rogl@oeaw.ac.at

Abbildungsnachweis: Taf. 1-3: Zeichnung Verf.; Abb. 1-10. 12. 14. 16-17: ÖAI Wien, Photo N. Gail, InvNr. EAG 02/030. EAG 02/038. EAG 02/032. EAG 00/343. EAG 02/034. EAG 02/026. EAG 01/207. EAG 01/211. EAG 02/048. EAG 02/041. EAG 02/042. EAG 99/357. EAG 01/165. EAG 02/046; Abb. 11. 13. 15. 18: Photo Verf. 